\title{
Internal time formalism for spacetimes with two Killing vectors
}

\author{
Joseph D. Romano \\ Department of Physics, University of Utah, Salt Lake City, Utah 84112 \\ and Department of Physics, University of Wisconsin, Milwaukee, Wisconsin 53201* \\ Charles G. Torre \\ Department of Physics, Utah State University, Logan, Utah 84322-4415
}

(Received 3 October 1995)

\begin{abstract}
The Hamiltonian structure of spacetimes with two commuting Killing vector fields is analyzed for the purpose of addressing the various problems of time that arise in canonical gravity. Two specific models are considered: (i) cylindrically symmetric spacetimes and (ii) toroidally symmetric spacetimes, which respectively involve open and closed universe boundary conditions. For each model, canonical variables which can be used to identify points of space and instants of time, i.e., internally defined spacetime coordinates, are identified. To do this it is necessary to extend the usual ADM phase space by a finite number of degrees of freedom. Canonical transformations are exhibited that identify each of these models with harmonic maps in the parametrized field theory formalism. The identifications made between the gravitational models and harmonic map field theories are completely gauge invariant; that is, no coordinate conditions are needed. The degree to which the problems of time are resolved in these models is discussed. [S0556-2821(96)02510-6]
\end{abstract}

PACS number(s): 04.60.Ds, 04.20.Ex, 04.60.Kz

\section{INTRODUCTION}

Throughout a large part of the history of general relativity, much effort has been expended toward disentangling the true degrees of freedom of the gravitational field from the "pure gauge", degrees of freedom brought into the theory via the principle of general covariance. In the Hamiltonian form of the theory, this problem involves understanding the solution space of the initial value constraints and the appropriate free data for the Cauchy problem. Classically, a characterization of the true degrees of freedom is relevant for analyzing the dynamical evolution of strongly gravitating systems, e.g., binary black hole systems, as well as for understanding fundamental issues in relativity, e.g., cosmic censorship. The intertwining of gauge degrees of freedom and dynamical degrees of freedom is especially vexing in quantum gravity, where it leads to many of the "problems of time" [1]. Evidently, canonical quantization of the gravitational field would be expediated by a sufficiently explicit characterization of the true degrees of freedom. For the most part, the strategy for doing this stems from the original Hamiltonian description of gravitation provided by Arnowitt, Deser, and Misner (ADM) [2] and Dirac [3]. The philosophy adopted there is that the Einstein field equations define an "already parametrized field theory" in which certain nondynamical canonical variables represent points of space and instants of time, relative to which the true degrees of freedom evolve. In the ADM approach, the dynamical content of relativity is exposed by coordinate conditions which fix the nondynamical gauge variables. This leads to a description of gravitational dynamics relative to a fixed foliation of spacetime, that is, relative to a fixed family of observers. A prominent example of this approach appears in the conformal ap-

\footnotetext{
* Current address.
}

proach to the initial value problem [4]. From such work it seems that the ADM approach is quite adequate for addressing many problems in classical relativity. However, the price paid for obtaining technical control over the true degrees of freedom is that general covariance is lost in the sense that one is obliged to view dynamics from the point of view of a given set of observers. In quantum theory this provides a first instance of a problem of time, which might be called the problem of general covariance: how to give the state of the gravitational field on an arbitrary hypersurface, that is, with respect to arbitrary observers. In the ADM approach one is prohibited from even asking this question.

An alternative approach to describing the true degrees of freedom of the gravitational field that preserves general covariance is available. This approach still relies upon the possibility of extracting "many-fingered time" degrees of freedom-or, more precisely, spacelike embeddings of Cauchy surfaces-from the gravitational phase space, but does not fix the foliation with coordinate conditions. Instead, one describes evolution of the true degrees of freedom relative to an arbitrary foliation; i.e., one casts the Einstein equations in the form of a parametrized field theory [5]. This point of view was developed in considerable detail by $\mathrm{Ku}$ char [6], who called it the "bubble time" dynamics of the gravitational field. Many of the problems of time are mitigated using this approach [1], which is now known as the "internal time formalism.' Implementation of the internal time formalism hinges upon the possibility of (i) finding a canonical transformation on the gravitational phase space which separates four canonical variables to play the role of spacelike embeddings of a Cauchy surface into spacetime and (ii) eliminating the momenta conjugate to the embeddings by the initial value constraints. If this can be done, the remaining variables represent the true degrees of freedom, whose dynamical evolution occurs via correlation with the arbitrary spacelike slices provided by the embedding vari- 
ables. So far, the internal time approach to gravitational dynamics has been implemented in lower-dimensional models, typically symmetry reductions of the full theory. There is a plethora of homogeneous cosmological models in which it is possible, at least locally, to isolate a canonical variable representing time and then solve the constraints for the momentum conjugate to time. These models possess a finite number of degrees of freedom. Relatively few field theoretic models, i.e., models possessing an infinity of degrees of freedom, exist in which one can implement the internal time program. Such models are, to our knowledge, always two-dimensional field theories, most notably Einstein-Rosen waves [7], planegravitational waves [8], spherically symmetric vacuum gravity [9], the bosonic string [10,11], and related models [12]. For the most part, these models are generally covariant theories of one or more free fields; e.g., the dynamics of the Einstein-Rosen wave is that of a single free scalar field representing the metric amplitude of the wave.

Our goal in this paper is to establish an internal time formalism for the reduction of the vacuum Einstein equations obtained by assuming the existence of two commuting spacelike Killing vector fields. We consider models involving both open universe and closed universe boundary conditions. Our work generalizes previous models, in particular the Einstein-Rosen waves [7], in two key ways. First, the reduced system of equations describes a pair of interacting fields. To our knowledge, this is the only symmetry reduction of the vacuum Einstein equations known that admits an internal time formulation and in which the true degrees of freedom constitute a nonlinear field theory. Second, in terms of the two Killing vector model, we illustrate in detail the fact that, strictly speaking, general relativity is not an already parametrized field theory. In order to extract canonical variables representing embeddings from the ADM phase space, it is necessary to extend that phase space by a finite number of degrees of freedom. For open universes, the additional nongravitational variables represent the asymptotic location of a spacelike hypersurface relative to an inertial frame "at infinity" [13]. For closed universes, the intrinsic and extrinsic geometries of a hypersurface are inadequate to define the embedding of that hypersurface in spacetime [14]; additional nongravitational variables are needed to accomplish this. Given the extensions of the phase space, the resulting dynamical system is in each case a generally covariant (parametrized) formulation of harmonic maps from a flat threedimensional spacetime to a two-dimensional target space of constant negative curvature.

In the next section we define the models. Our open universe model is obtained by imposing cylindrical symmetry on spacetime. Our closed universe model, first considered by Gowdy [15], defines the universe to be a three-torus which admits a two-torus group of isometries. We do not impose hypersurface orthogonality (reflection symmetry) on the Killing vectors, and the resulting dynamical theory is in each case intrinsically nonlinear, in contrast to the Einstein-Rosen waves [7] or "polarized" Gowdy models [16]. In the language of the linearized theory, both gravitational polarizations propagate and interact. In Sec. III we exhibit canonical transformations, inspired by [7] and [10], which allow us to extract the embeddings, solve the constraints, and reveal the true degrees of freedom in each model. Other two Killing vector models, which differ in the choice of isometry group, spacetime topology, etc., can be treated in much the same way as we do here. As mentioned above, these results lead to substantial simplifications in the problems of time that occur in canonical quantum gravity. We discuss these problems and the general structure of the quantum theory based on the internal time formulation of the two Killing vector models in Sec. IV. In the cylindrically symmetric model, it is necessary to keep track of the behavior of fields on the axis of symmetry and at infinity. We summarize our boundary and falloff conditions in the Appendix, which is referred to throughout the paper.

\section{SPACETIMES WITH TWO COMMUTING KILLING VECTORS}

We are going to study the Hamiltonian structure of spacetimes $(\mathscr{l}, g)$ which admit two linearly independent spacelike Killing vector fields $K_{A}^{\alpha}, A=1,2$. The Killing vector fields are assumed to commute,

$$
\left[K_{A}, K_{B}\right]^{\alpha}=0,
$$

and to generate a two-dimensional "orthogonally transitive" group $\mathscr{G}$ of isometries. This latter requirement means that the distribution of two-dimensional vector spaces spanned by $K_{A}^{\alpha}$ at each point is orthogonal to a foliation of $\mathscr{C} 6$ by surfaces $M \hookrightarrow \mathscr{C l}$. Our assumptions amount to demanding that the integral curves of the Killing vector fields provide a fibration $\pi: \mathscr{L} \rightarrow M$ of the spacetime manifold by spacelike surfaces. The two-dimensional manifold $M$ is the "space of orbits" of the Killing vector fields.

The spacetime metric $g_{\alpha \beta}$ defines three functions

$$
\lambda_{A B}=g_{\alpha \beta} K_{A}^{\alpha} K_{B}^{\beta},
$$

representing the lengths and inner products of the Killing vector fields. Because the Killing vectors are spacelike and linearly independent at each point, this symmetric matrix will have a symmetric inverse $\lambda^{A B}$. We can use $\lambda^{A B}$ to define a projection operator $\gamma_{\beta}^{\alpha}$ into the (co)tangent space to each point of $M$ :

$$
\gamma_{\beta}^{\alpha}=\delta_{\beta}^{\alpha}-\lambda^{A B} K_{A}^{\alpha} K_{B \beta} .
$$

The associated tensor field

$$
\gamma_{\alpha \beta}=g_{\alpha \beta}-\lambda^{A B} K_{A \alpha} K_{B \beta}
$$

satisfies

$$
\mathscr{b}_{K_{A}} \gamma_{\alpha \beta}=0 \quad \text { and } K_{A}^{\alpha} \gamma_{\alpha \beta}=0 \text {. }
$$

There is a one-to-one correspondence between tensor fields on $M$ and tensor fields on $\mathscr{C l}$ with vanishing Lie derivatives along $K_{A}^{\alpha}$ and which are completely "orthogonal" to $K_{A}^{\alpha}$ $[17,18]$. This correspondence means that $\gamma_{\alpha \beta}$ uniquely defines a Lorentz metric on $M$.

Coordinates $x^{\alpha}=\left(x^{A}, x^{a}\right), A=1,2, a=3,4$, can be chosen such that

$$
K_{A}^{\alpha}=\delta_{A}^{\alpha},
$$

and the spacetime metric takes the form 


$$
g_{\alpha \beta} d x^{\alpha} d x^{\beta}=\lambda_{A B} d x^{A} d x^{B}+\gamma_{a b} d x^{a} d x^{b},
$$

where $\lambda_{A B}=\lambda_{A B}\left(x^{a}\right)$ and $\gamma_{a b}=\gamma_{a b}\left(x^{a}\right)$.

There are only six independent Einstein equations because the orthogonal transitivity requirement forces

$$
\gamma_{\gamma}^{\alpha} K_{A}^{\beta} G_{\alpha \beta} \equiv 0 .
$$

Thus the nontrivial Einstein equations can be taken to be

$$
\begin{gathered}
K_{A}^{\alpha} K_{B}^{\beta} G_{\alpha \beta}=0, \\
\gamma_{\gamma}^{\alpha} \gamma_{\delta}^{\beta} G_{\alpha \beta}=0 .
\end{gathered}
$$

Setting $\lambda=\operatorname{det}\left(\lambda_{A B}\right)$, these equations can be put into the following form in the coordinates $\left(x^{A}, x^{a}\right)$ :

$$
\begin{gathered}
-\frac{1}{2} \lambda^{1 / 2} D^{a}\left(\lambda^{-1 / 2} D_{a} \lambda_{A B}\right)+\lambda_{A B}\left(-\frac{1}{2} \mathscr{B}+\lambda^{-1 / 2} D^{a} D_{a} \lambda^{1 / 2}\right. \\
\left.+\frac{3}{2} D^{a} \lambda^{-1 / 2} D_{a} \lambda^{1 / 2}-\frac{3}{8} D^{a} \lambda^{C D} D_{a} \lambda_{C D}\right)=0, \\
\frac{1}{4} D_{c} \lambda^{A B} D_{d} \lambda_{A B}-D_{c}\left(\lambda^{-1 / 2} D_{d} \lambda^{1 / 2}\right)+\gamma_{c d}\left(\lambda^{-1 / 2} D^{a} D_{a} \lambda^{1 / 2}\right. \\
\left.+\frac{1}{2} D^{a} \lambda^{-1 / 2} D_{a} \lambda^{1 / 2}-\frac{1}{8} D^{a} \lambda^{A B} D_{a} \lambda_{A B}\right)=0 .
\end{gathered}
$$

Here $D_{a}$ is the derivative operator on $M$ compatible with $\gamma_{a b}$ and $\mathscr{B}$ is the scalar curvature of $D_{a}$. Indices for tensor fields on $M$ (Latin lowercase) are lowered and raised with $\gamma_{a b}$ and its inverse $\gamma^{a b}$. It will be useful later to note that Eq. (2.11) implies

$$
D^{a} D_{a}\left(\lambda^{1 / 2}\right)=0 .
$$

All of our considerations will be formulated on the space of orbits $M$. The Einstein equations (2.10) and (2.11) are viewed as a generally covariant system of partial differential equations for "matter fields" $\lambda_{A B}$ and Lorentz metric $\gamma_{a b}$ on the two-dimensional manifold $M$.

\section{A. Cylindrical symmetry}

Our model for open universes is defined by taking $\mathscr{C}=\mathbf{R}^{3} \times \mathbf{S}^{1}$ and $\mathscr{G}=\mathbf{S}^{1} \times \mathbf{R}$. Note that $\mathscr{C}$ is diffeomorphic to the manifold obtained by removing a timelike two-plane (swept out by, say, the $z$ axis) from $\mathbf{R}^{4}$. The Killing coordinates are denoted by $x^{A}=(\phi, z)$, where $\phi \in(0,2 \pi)$ and $z$ $\in(-\infty, \infty)$. The space of orbits is $M=\mathbf{R} \times \mathbf{R}^{+}$, where $\mathbf{R}^{+}$is the manifold of positive-definite real numbers. We define $R:=\lambda^{1 / 2}$ and demand that $\boldsymbol{\nabla}_{\alpha} R$ is everywhere spacelike. We will call spacetimes with these properties cylindrically symmetric. In what follows it will be useful to employ coordinates on $M$ that are adapted to a foliation by spacelike curves $\mathbf{R}^{+} \hookrightarrow M$. These coordinates will be denoted by $x^{a}=(t, r)$ where $t \in(-\infty, \infty)$ and $r \in(0, \infty)$. The radial variable $r$ will be restricted by the requirement that $\partial R / \partial r>0$. $R$ itself can serve as a radial coordinate on $\mathbf{R}^{+}$. Because of (2.12), $R$ will be a harmonic coordinate when the Einstein equations hold. The harmonic conjugate $T$ to $R$ is defined by

$$
D_{a} T=\epsilon_{a}^{b} D_{b} R,
$$

where $\epsilon_{a b}$ is the volume form on $M$ defined by $\gamma_{a b}$. The coordinates $t=T$ and $r=R$ form a conformal coordinate chart when the Einstein equations hold; that is, in these coordinates the metric on $M$ is given by

$$
d s^{2}=\Omega^{2}\left(-d T^{2}+d R^{2}\right),
$$

where $\Omega=\Omega(T, R)$ is a positive-definite function.

We parametrize $\lambda_{A B}$ in terms of scalar fields $(R, \psi, \widetilde{\psi})$ via

$$
\lambda_{A B} d x^{A} d x^{B}=R^{2} e^{-\psi} d \phi^{2}+e^{\psi}(d z+\widetilde{\psi} d \phi)^{2} .
$$

In preparation for the Hamiltonian formulation of the Einstein equations (2.10) and (2.11), we foliate $M$ with spacelike curves $t=$ const and parametrize the metric on $M$ via

$$
\begin{aligned}
\gamma_{a b} d x^{a} d x^{b}= & {\left[-\left(N^{\perp}\right)^{2}+e^{\gamma-\psi}\left(N^{r}\right)^{2}\right] d t^{2}+2 e^{\gamma-\psi} N^{r} d t d r } \\
& +e^{\gamma-\psi} d r^{2}
\end{aligned}
$$

As tensor fields on the curves $t=$ const, $N^{\perp}$ is a scalar field called the "lapse function," $N^{r}$ is a vector field called the "shift vector" (equivalent to a density of weight minus one on $\mathbf{R}^{+}$), and $\gamma$ is the natural logarithm of a covariant rank-2 tensor field (equivalent to the logarithm of a density of weight two). The functions $(R, \psi, \widetilde{\psi})$ are scalar fields on $t=$ const. All the fields $\left(N^{\perp}, N^{r}, \gamma, R, \psi, \widetilde{\psi}\right)$ are functions of the coordinates $(t, r)$ only; see the Appendix for the boundary and falloff conditions we use for these fields. In terms of this parametrization, the conformal factor $\Omega$ arising in the coordinates $x^{a}=(T, R)$ [see (2.14)] is given by

$$
\Omega=e^{(\gamma-\psi) / 2} .
$$

It is worth noting some special cases of the parametrization given above. If the Killing vectors are each hypersurface orthogonal, it follows that $\widetilde{\psi}=0$; i.e., the matrix $\lambda_{A B}$ is diagonal, indicating orthogonality of the Killing vectors. This special case of cylindrical symmetry in which each $K_{A}^{\alpha}$ is hypersurface orthogonal will be called whole cylindrical symmetry. The solutions to the field equations possessing whole cylindrical symmetry are the well-known EinsteinRosen waves. Kuchar assumes whole cylindrical symmetry in his analysis [7], and, with $\widetilde{\psi}=0$, our parametrization of the spacetime metric is identical to his. When

$$
N^{\perp}=1, \quad N^{r}=0, \quad \gamma=0, \quad R=r, \quad \psi=0, \quad \widetilde{\psi}=0,
$$

the spacetime is flat and the metric is expressed in cylindrical coordinates.

Let an overdot denote differentiation with respect to $t$. The field equations (2.10) and (2.11) in the parametrization (2.15) and (2.16) can be obtained from the following Hamiltonian form of the action:

$$
\begin{aligned}
& S\left[N^{\perp}, N^{r}, \gamma, R, \psi, \widetilde{\psi}, \pi_{\gamma}, \pi_{R}, \pi_{\psi}, \pi_{\tilde{\psi}}\right] \\
& \quad=\int_{t_{1}}^{t_{2}} d t \int_{0}^{\infty} d r\left(\pi_{\gamma} \dot{\gamma}+\pi_{R} \dot{R}+\pi_{\psi} \dot{\psi}+\pi_{\widetilde{\psi}} \dot{\tilde{\psi}}\right)-\int_{t_{1}}^{t_{2}} d t \mathbf{H},
\end{aligned}
$$

where the Hamiltonian is 


$$
\mathbf{H}=2 N^{\perp}(\infty)\left(1-e^{-\gamma(\infty) / 2}\right)+\int_{0}^{\infty} d r\left(N^{\perp} \mathscr{H}_{\perp}+N^{r} \mathscr{H}_{r}\right),
$$

and we have denoted the limits of $N^{\perp}(r)$ and $\gamma(r)$ as $r \rightarrow \infty$ by $N^{\perp}(\infty)$ and $\gamma(\infty)$. The super-Hamiltonian and supermomentum are given by

$$
\begin{aligned}
\mathscr{H}_{\perp}:= & e^{(\psi-\gamma) / 2}\left[-\pi_{\gamma} \pi_{R}+2 R^{\prime \prime}-R^{\prime} \gamma^{\prime}\right. \\
& +\frac{1}{2}\left(R \psi^{\prime 2}+R^{-1} \pi_{\psi}^{2}\right)+\frac{1}{2}\left(R e^{-2 \psi} \pi_{\widetilde{\psi}}^{2}\right. \\
& \left.\left.+R^{-1} e^{2 \psi} \widetilde{\psi}^{\prime 2}\right)\right], \\
\mathscr{H}_{r}:= & -2 \pi_{\gamma}^{\prime}+\pi_{\gamma} \gamma^{\prime}+\pi_{R} R^{\prime}+\pi_{\psi} \psi^{\prime}+\pi_{\widetilde{\psi}} \widetilde{\psi^{\prime}},
\end{aligned}
$$

where a prime indicates a derivative with respect to $r$. For each $t$ the momentum variables $\left(\pi_{\gamma}, \pi_{R}, \pi_{\psi}, \pi_{\psi}\right)$ are scalar densities of weight one on $\mathbf{R}^{+}$. The Hamiltonian action functional (2.19) can be obtained by (i) expressing the ADM action in terms of the parametrization (2.15) and (2.16), (ii) integrating out the Killing coordinates $z$ and $\phi$ (the range of $z$ should be made finite), and (iii) dividing the result by the range of $z$ and the range of $\phi$.

Extremizing the action (2.19) with respect to variations of $\pi_{\gamma}, \pi_{R}, \pi_{\psi}$, and $\pi_{\tilde{\psi}}$ produces the definitions of the momenta in terms of the "velocities" $\dot{\gamma}, \dot{R}, \dot{\psi}$, and $\tilde{\tilde{\psi}}$. Extremizing the action (2.19) with respect to variations of $N^{\perp}, N^{r}, \gamma, R, \psi$, and $\widetilde{\psi}$, where the asymptotic values of $N^{\perp}$ and $N^{r}$ are held fixed, yields six equations, which, given the definitions of the momenta, are equivalent to the six field equations (2.10) and (2.11) in the parametrization (2.15) and (2.16). In particular, the field equations arising from varying the lapse and shift are the constraints

$$
\mathscr{H}_{\perp} \approx 0 \text { and } \mathscr{H}_{r} \approx 0,
$$

which correspond to the normal-normal and normaltangential projections of the field equations (2.11) relative to the curve $t=$ const. The remaining four equations are evolution equations for the spatial metric variable $\gamma$ and the "matter fields" $R, \psi$, and $\psi$.

Let us make two remarks on the Hamiltonian variational principle we have described. (i) It is important to note that the canonical variables are restricted by the assumption that $\nabla_{\alpha} R$ is spacelike on $\mathscr{C}$, which implies that $D_{a} R$ is spacelike on $M$. In Hamiltonian form, this restriction is

$$
R^{\prime}>\left|\pi_{\gamma}\right|
$$

(ii) The boundary term at infinity that appears in the Hamiltonian is needed to render the action and Hamiltonian functionally differentiable with the boundary conditions given in the Appendix. On solutions to the field equations, the Hamiltonian is given by the boundary term, which we identify as the energy that generates time evolution at infinity characterized by $N^{\perp}(\infty)$. Note that our boundary conditions are such that $N^{\perp}(\infty)$ is fixed, that is, not subject to variation. This allows us to add constant multiples of $N^{\perp}(\infty)$ to the surface term without spoiling the differentiability of the action or Hamiltonian. We have used this freedom to normalize the Hamiltonian so that it vanishes when spacetime is flat, which occurs when $\gamma(\infty)=0$. The energy associated with time translations described by unit lapse function at infinity is

$$
E=2\left(1-e^{-\gamma(\infty) / 2}\right)
$$

It follows from the field equations that $\gamma(\infty)$, known as the " $C$ energy," is conserved and non-negative (see, e.g., [19]). Hence $E$ is bounded from below and is minimized on flat spacetime. Note also that $E$ is bounded from above, which is due to the geometric interpretation of $E$ as a deficit angle (divided by $\pi$ ) at infinity. See [20] for a detailed discussion of this interesting behavior of the energy of cylindrically symmetric spacetimes.

To summarize, cylindrically symmetric spacetimes constitute a constrained Hamiltonian system. The phase space $\Gamma$ is the space of fields $\left(\gamma, R, \psi, \widetilde{\psi}, \pi_{\gamma}, \pi_{R}, \pi_{\psi}, \pi_{\widetilde{\psi}}\right)$ on $\mathbf{R}^{+}$with boundary conditions as specified in the Appendix and with the restriction (2.24). The action functional (2.19) defines the symplectic two-form $\Omega$ on $\Gamma$. $\Omega$ maps a pair of tangent vectors

$$
X=\left(\delta \gamma, \delta R, \delta \psi, \delta \widetilde{\psi}, \delta \pi_{\gamma}, \delta \pi_{R}, \delta \pi_{\psi}, \delta \pi_{\tilde{\psi}}\right)
$$

and

$$
\hat{X}=\left(\hat{\delta} \gamma, \hat{\delta} R, \hat{\delta} \psi, \hat{\delta} \widetilde{\psi}, \hat{\delta} \pi_{\gamma}, \hat{\delta} \pi_{R}, \hat{\delta} \pi_{\psi}, \hat{\delta} \pi_{\tilde{\psi}}\right)
$$

to the real number

$$
\begin{aligned}
\Omega(X, \hat{X})= & \int_{0}^{\infty} d r\left(\delta \pi_{\gamma} \hat{\delta} \gamma+\delta \pi_{R} \hat{\delta} R+\delta \pi_{\psi} \hat{\delta} \psi+\delta \pi_{\psi} \hat{\delta} \widetilde{\psi}\right. \\
& -[\delta \leftrightarrow \hat{\delta}]) .
\end{aligned}
$$

From (2.28) it follows that $(\gamma, R, \psi, \widetilde{\psi})$ and $\left(\pi_{\gamma}, \pi_{R}, \pi_{\psi}, \pi_{\tilde{\psi}}\right)$ are, respectively, canonical coordinates and momenta for $\Gamma$ and hence satisfy the canonical Poisson brackets relations, e.g.,

$$
\left\{\gamma(r), \pi_{\gamma}(\vec{r})\right\}=\delta(r, \vec{r}) .
$$

In terms of the Poisson bracket $\{\cdot, \cdot\}$, the time evolution of a function $F: \Gamma \rightarrow \mathbf{R}$ is given by

$$
\dot{F}=\{F, \mathbf{H}\} .
$$

For any choice of lapse and shift, dynamical evolution takes place entirely on the constraint surface $\bar{\Gamma} \hookrightarrow \Gamma$ defined by the constraints (2.23). This follows from the fact that the constraints are "first class." More precisely, the Poisson algebra of the super-Hamiltonian and supermomentum is the algebra of deformations of spacelike curves in $M$ [21].

In two-dimensional spacetimes, such as we have here, it is convenient to work with a rescaled super-Hamiltonian which is a scalar density of weight two. We define the weight-two super-Hamiltonian via

$$
\mathscr{H}=e^{(\gamma-\psi) / 2} \mathscr{H}_{\perp} .
$$


Of course, the super-Hamiltonian constraint $\mathscr{H}_{\perp} \approx 0$ is equivalent to $\mathscr{H} \approx 0$, and we can write

$$
N^{\perp} \mathscr{H}_{\perp}=N \mathscr{H}
$$

where $N$ is a scalar density of weight minus one, which is equivalent to a vector in one dimension, obtained by

$$
N=e^{(\psi-\gamma) / 2} N^{\perp}
$$

We can now vary the action

$$
\begin{aligned}
\bar{S}\left[N, N^{r}, \gamma, R, \psi, \widetilde{\psi}, \pi_{\gamma}, \pi_{R}, \pi_{\psi}, \pi_{\psi}\right] \\
=\int_{t_{1}}^{t_{2}} d t \int_{0}^{\infty} d r\left(\pi_{\gamma} \dot{\gamma}+\pi_{R} \dot{R}+\pi_{\psi} \dot{\psi}+\pi_{\psi} \tilde{\dot{\psi}}-N \mathscr{H}\right. \\
\left.\quad-N^{r} \mathscr{H}_{r}\right)-\int_{t_{1}}^{t_{2}} d t N(\infty) \gamma(\infty)
\end{aligned}
$$

with respect to its arguments and obtain equations still equivalent to (2.10) and (2.11) once the correspondence (2.33) is made. In this variational principle we hold fixed the asymptotic value of $N$. When using the weight-two superHamiltonian and associated lapse density, the Hamiltonian is given by

$$
\overline{\mathbf{H}}=N(\infty) \gamma(\infty)+\int_{0}^{\infty} d r\left(N \mathscr{H}+N^{r} \mathscr{H}_{r}\right)
$$

where the surface term is again chosen to make $\bar{S}$ and $\overline{\mathbf{H}}$ differentiable with our boundary conditions and to yield $\overline{\mathbf{H}}=0$ when $\gamma(\infty)=0$. Evidently, the $C$ energy is associated with time translations defined by unit lapse density at infinity.

The advantage of the weight-two super-Hamiltonian is that the Poisson algebra of constraints is now a Lie algebra [22]. In detail, define the smeared constraints

$$
\mathscr{H}(N)=\int_{0}^{\infty} d r N \mathscr{H} \quad \text { and } \mathscr{H}_{r}\left(N^{r}\right)=\int_{0}^{\infty} d r N^{r} \mathscr{H}_{r},
$$

where $N^{r}$ is a given vector field and $N$ is a given scalar density of weight minus one. Direct computation then shows that

$$
\begin{gathered}
\{\mathscr{H}(N), \mathscr{H}(M)\}=\mathscr{H}_{r}\left(J^{r}\right), \\
\left\{\mathscr{H}(N), \mathscr{H}_{r}\left(M^{r}\right)\right\}=\mathscr{H}(K), \\
\left\{\mathscr{H}_{r}\left(N^{r}\right), \mathscr{H}_{r}\left(M^{r}\right)\right\}=\mathscr{H}_{r}\left(L^{r}\right),
\end{gathered}
$$

where

$$
\begin{gathered}
J^{r}=N M^{\prime}-M N^{\prime}, \\
K=N M^{r \prime}-M^{r} N^{\prime}, \\
L^{r}=N^{r} M^{r \prime}-M^{r} N^{r \prime} .
\end{gathered}
$$

\section{B. Toroidal symmetry}

Our model for closed universes is obtained by setting $\mathscr{C}=\mathbf{R}^{+} \times \mathbf{T}^{3}$ and choosing the isometry group to be $\mathscr{G}=\mathbf{T}^{2}$ with coordinates $y, z \in(0,2 \pi)$. The space of orbits is a cylinder, $M=\mathbf{R}^{+} \times \mathbf{S}^{1}$. We are considering one of the Gowdy models [15], and we shall call spacetimes with the above properties toroidally symmetric. In these spacetimes we define $\tau:=\lambda^{1 / 2}$. It can be shown that toroidally symmetric vacuum spacetimes are foliated by spacelike surfaces whose leaves are defined by $\tau=$ const $[15,23]$. Hence the gradient of $\tau$ is timelike, which we will assume in all that follows. According to observers "at rest" relative to the slices $\tau=$ const, the toroidally symmetric spacetimes expand forever from a " "big bang" at $\tau=0$. Coordinates on $M$ that are adapted to a foliation by spacelike circles will be denoted $(t, x)$, where $t \in(0, \infty)$ and $x \in(-\infty, \infty)$ with the identification $x \sim x+2 \pi$. We will demand that a time coordinate $t$ on $M$ satisfy $\partial \tau / \partial t$ $>0$. For any solution of the Einstein equations, $\tau$ is a harmonic time coordinate. The harmonic conjugate to $\tau$ is denoted by $X$, which satisfies

$$
D_{a} X=\frac{\epsilon_{a}^{b} D_{b} \tau}{\frac{1}{2 \pi} \int_{\mathbf{s}^{1}} d s^{a} \epsilon_{a}{ }^{b} D_{b} \tau},
$$

where $d s^{a}$ is the oriented line element on a spacelike circle. Because $D_{a} \tau$ is timelike, the denominator in (2.39) never vanishes. By virtue of (2.12), the integral in the denominator is independent of the choice of spacelike circle, i.e., is a constant of motion. This integral is introduced so that on any circle $t=$ const we have that

$$
X(x+2 \pi)-X(x)=2 \pi .
$$

With the identification $X \sim X+2 \pi$, the coordinates $t=\tau$, $x=X$ are adapted to a spacelike foliation of $M$; in these coordinates, the metric on $M$ is of the form

$$
d s^{2}=\Omega^{2}\left(-d \tau^{2}+d X^{2}\right) .
$$

The Hamiltonian formulation of toroidally symmetric spacetimes closely parallels that obtained for cylindrically symmetric spacetimes. Essentially, the toroidally symmetric case differs by the change of notation $R \leftrightarrow \tau$ and the fact that the Cauchy surfaces in $M$ are now compact circles parametrized by $x$ instead of noncompact half-lines parametrized by $r$. We parametrize $\lambda_{A B}$ in terms of scalar fields $(\tau, \psi, \widetilde{\psi})$ via

$$
\lambda_{A B} d x^{A} d x^{B}=\tau^{2} e^{-\psi} d y^{2}+e^{\psi}(d z+\widetilde{\psi} d y)^{2} .
$$

The metric on $M$ is parametrized relative to an arbitrary foliation by spacelike circles exactly as in (2.16):

$$
\begin{aligned}
\gamma_{a b} d x^{a} d x^{b}= & {\left[-\left(N^{\perp}\right)^{2}+e^{\gamma-\psi}\left(N^{x}\right)^{2}\right] d t^{2}+2 e^{\gamma-\psi} N^{x} d t d x } \\
& +e^{\gamma-\psi} d x^{2} .
\end{aligned}
$$

In the coordinates $x^{a}=(\tau, X)$ the conformal factor in $(2.41)$ is

$$
\Omega=e^{(\gamma-\psi) / 2} .
$$

When $\widetilde{\psi}=0$ our parametrization corresponds to a "polarized Gowdy model" [16], which can be considered the closed universe analog of the Einstein-Rosen waves. When 


$$
N^{\perp}=1, \quad N^{x}=0, \quad \gamma=0, \quad \tau=t, \quad \psi=0, \quad \widetilde{\psi}=0,
$$

the spacetime is flat. This spacetime can be obtained from four-dimensional Minkowski spacetime $\mathbf{M}^{4}$ as follows. Let $(\hat{t}, \hat{x}, \hat{y}, \hat{z})$ be inertial coordinates on $\mathbf{M}^{4}$. Denote by $I$ the submanifold of $\mathbf{M}^{4}$ in which $\hat{t}^{2}-\hat{y}^{2}>0$ and $\hat{t}>0$. Let $\widetilde{I}$ denote the manifold obtained from $I$ by the identifications

$$
\begin{gathered}
\hat{x} \sim \hat{x}+2 \pi, \\
\operatorname{arctanh}\left(\frac{\hat{y}}{\hat{t}}\right) \sim \operatorname{arctanh}\left(\frac{\hat{y}}{\hat{t}}\right)+2 \pi, \\
\hat{z} \sim \hat{z}+2 \pi .
\end{gathered}
$$

The Minkowski metric $\eta_{\alpha \beta}$ projects to a flat metric on $\widetilde{I}$. The mapping $\phi: \mathscr{l} \rightarrow \widetilde{I}$, defined by

$$
\begin{gathered}
\hat{t}=t \cosh y, \\
\hat{x}=x, \\
\hat{y}=t \sinh y, \\
\hat{z}=z,
\end{gathered}
$$

is a diffeomorphism which identifies the metric on $\mathscr{C l}$ defined by (2.45),

$$
d s^{2}=-d t^{2}+d x^{2}+t^{2} d y^{2}+d z^{2},
$$

with the flat metric induced on $\widetilde{I}$.

In terms of a spacelike foliation of $M$ with adapted coordinates $(t, x)$, the Hamiltonian form of the action is

$$
\begin{aligned}
& S\left[N^{\perp}, N^{r}, \gamma, \tau, \psi, \widetilde{\psi}, \pi_{\gamma}, \pi_{\tau}, \pi_{\psi}, \pi_{\tilde{\psi}}\right] \\
& \quad=\int_{t_{1}}^{t_{2}} d t \int_{0}^{2 \pi} d x\left(\pi_{\gamma} \dot{\gamma}+\pi_{\tau} \dot{\tau}+\pi_{\psi} \dot{\psi}+\pi_{\tilde{\psi}} \dot{\tilde{\psi}}\right)-\int_{t_{1}}^{t_{2}} d t \mathbf{H},
\end{aligned}
$$

where the Hamiltonian is

$$
\mathbf{H}=\int_{0}^{2 \pi} d x\left(N^{\perp} \mathscr{H}_{\perp}+N^{x} \mathscr{H}_{x}\right)
$$

The super-Hamiltonian and supermomentum are given by

$$
\begin{aligned}
& \mathscr{H}_{\perp}:= e^{(\psi-\gamma) / 2}\left[-\pi_{\gamma} \pi_{\tau}+2 \tau^{\prime \prime}-\tau^{\prime} \gamma^{\prime}+\frac{1}{2}\left(\tau \psi^{\prime 2}+\tau^{-1} \pi_{\psi}^{2}\right)\right. \\
&\left.+\frac{1}{2}\left(\tau e^{-2 \psi} \pi_{\widetilde{\psi}}^{2}+\tau^{-1} e^{2 \psi} \widetilde{\psi}^{\prime 2}\right)\right] \approx 0, \\
& \mathscr{H}_{x}:=-2 \pi_{\gamma}^{\prime}+\pi_{\gamma} \gamma^{\prime}+\pi_{\tau} \tau^{\prime}+\pi_{\psi} \psi^{\prime}+\pi_{\widetilde{\psi}} \widetilde{\psi}^{\prime} \approx 0,
\end{aligned}
$$

where a prime denotes differentiation with respect to $x$. As in the cylindrically symmetric case, the action functional (2.49) can be obtained by expressing the usual ADM action in the field parametrization (2.42) and (2.43), integrating out the coordinates $y$ and $z$, and dividing by the ranges of these coordinates. Extremizing (2.49) with respect to variations of its arguments leads to the Einstein field equations (2.10) and (2.11) in our chosen parametrization.

To summarize, points in the phase space $\Gamma$ of toroidally symmetric spacetimes are defined by the smooth tensor fields on the circle $\left(\gamma, \tau, \psi, \widetilde{\psi}, \pi_{\gamma}, \pi_{\tau}, \pi_{\psi}, \pi_{\widetilde{\psi}}\right)$. These fields are restricted by the requirement that $\nabla_{\alpha} \tau$ be timelike on $\mathscr{C}$, which means $D_{a} \tau$ is timelike on $M$; in Hamiltonian form, this requirement is

$$
\pi_{\gamma}<-\left|\tau^{\prime}\right|
$$

The action (2.49) defines the symplectic structure on $\Gamma$. The symplectic two-form acting on a pair of tangent vectors to $\Gamma$ is given by

$$
\begin{aligned}
\Omega(X, \hat{X})= & \int_{0}^{2 \pi} d x\left(\delta \pi_{\gamma} \hat{\delta} \gamma+\delta \pi_{\tau} \hat{\delta} \tau+\delta \pi_{\psi} \hat{\delta} \psi+\delta \pi_{\tilde{\psi}} \hat{\delta} \tilde{\psi}\right. \\
& -[\delta \leftrightarrow \hat{\delta}]),
\end{aligned}
$$

so that, with $R \leftrightarrow \tau$, the canonical coordinates and momenta are as before. The weight-two super-Hamiltonian is defined via (2.31), and the Poisson-algebraic properties of the constraint functions are as in the cylindrically symmetric case. Dynamical evolution takes place on the constraint surface $\bar{\Gamma}$ defined by the constraints (2.51) and (2.52), and is generated by the Hamiltonian (2.50).

\section{CANONICAL TRANSFORMATIONS}

In this section we will exhibit canonical transformations from slight extensions of the gravitational phase spaces of Sec. II to phase spaces for parametrized field theories on a fixed background spacetime. The extensions are needed because the gravitational phase space is not quite adequate to define embeddings of hypersurfaces into Ricci-flat spacetimes. This difficulty arises for the full theory in both closed universes [14] and open universes [13], and can be considered a "global problem of time" [1]. While it is possible to phrase all of our results directly on the four-dimensional spacetime manifold $\mathscr{C l}$, it is far more convenient to express our results on the space of orbits $M$, and we will present our analysis on this effective two-dimensional spacetime manifold.

Before specializing to our two Killing vector models, let us outline the basic strategy for the full theory $[6,1]$. Denote the usual gravitational phase space variables by $\left(q_{i j}, p^{i j}\right)$ and the super-Hamiltonian and super-momentum by $\left(\mathscr{H}_{\perp}, \mathscr{H}_{i}\right)$. We seek a canonical transformation

$$
\left(q_{i j}, p^{i j}\right) \rightarrow\left(X^{\alpha}, \Pi_{\alpha}, q^{\mathbf{A}}, p_{\mathbf{A}}\right),
$$

where $\alpha=0,1,2,3$ and $\mathbf{A}=1,2$ such that, on solutions to the equations of motion and constraints, $X^{\alpha}: \Sigma \rightarrow \mathscr{C l}$ represents a spacelike embedding of a Cauchy surface $\Sigma$ into the spacetime manifold $\mathscr{L}$. The transformation must allow the con- 
straints $\mathscr{H}_{\perp} \approx 0 \approx \mathscr{H}_{i}$ to be resolved for the momenta conjugate to the embeddings; i.e., in the new variables the constraints are equivalent to

$$
H_{\alpha}:=\Pi_{\alpha}+h_{\alpha}\left(X^{\alpha}, q^{\mathbf{A}}, p_{\mathbf{A}}\right) \approx 0 .
$$

This formulation of the canonical theory has the following interpretation. The gravitational variables encoded in $X^{\alpha}$ are used to identify instants of time and points of space at which the true degrees of freedom $\left(q^{\mathbf{A}}, p_{\mathbf{A}}\right)$ are being measured. The embeddings are "'pure gauge," i.e., arbitrary; their conjugate momenta are completely determined in terms of the embeddings and true degrees of freedom by the constraints (3.2). The constraint functions $H_{\alpha}$, when integrated against functions $N^{\alpha}$, generate the dynamical evolution of the true degrees of freedom $\left(q^{\mathbf{A}}, p_{\mathbf{A}}\right)$ as the embedding they are on is deformed, via $\delta X^{\alpha}=N^{\alpha}$, through the Ricci-flat spacetime for which $\left(q^{\mathbf{A}}, p_{\mathbf{A}}\right)$ are Cauchy data. The densities of weight-one $h_{\alpha}\left(X^{\alpha}, q^{\mathbf{A}}, p_{\mathbf{A}}\right)$ represent the energy-momentum current of $\left(q^{\mathbf{A}}, p_{\mathbf{A}}\right)$ through the hypersurface embedded by $X^{\alpha}$. Gravitational dynamics on phase space is thus cast into the form of a "parametrized field theory" on $\mathscr{C l}$.

\section{A. Open universes: Cylindrical symmetry}

As we saw in Sec. II A, the metric variable $R$ and its harmonic conjugate $T$ define a conformal coordinate chart on cylindrically symmetric spacetimes (reduced to $M$ ). We can thus define a spacelike curve $\mathbf{R}^{+} \hookrightarrow M$ by giving its parametric description $(T(r), R(r))$. In the canonical formalism we can therefore view the phase space variable $R(r)$ as one part of an embedding of $\mathbf{R}^{+}$into $M$. To complete the definition of the embedding, we must express $T$ as a function on the phase space, which will be again denoted $T(r)$. Using the pullback of (2.13) to a spacelike curve $t=$ const and the Hamilton equations, we find

$$
T^{\prime}=-\pi_{\gamma}
$$

This equation can be integrated to give

$$
T(r)=T(\infty)-\int_{\infty}^{r} d \bar{r} \pi_{\gamma}(\vec{r}) .
$$

Because of the restriction (2.24), the variables $(T(r), R(r))$ define a spacelike embedding on solutions of the equations of motion. Unfortunately, given a point in the gravitational phase space $\Gamma$, the embedding is not uniquely specified because the gravitational data do not fix the value of the integration constant $T(\infty)$, which represents the asymptotic location of the embedded curve. This difficulty is not a consequence of our use of the conformal coordinates $(T, R)$ to specify an embedding, but is a general feature of general relativity of open universes. For example, in [13] the Hamiltonian formulation of general relativity in asymptotically flat universes is considered, and it is shown that the geometrodynamical data must be supplemented by a finite number of additional degrees of freedom in order to determine a spacelike hypersurface. To implement this idea in the context of cylindrically symmetric spacetimes, we will extend the phase space $\Gamma$ of Sec. II A. We then make a canonical change of variables on the extended phase space in which certain de- grees of freedom are identified as spacelike embeddings. The resulting Hamiltonian structure of the model is then interpreted as a generally covariant form of a theory of harmonic maps by a sequence of canonical transformations on the appropriate harmonic map phase space. We begin by finding the appropriate extended phase space for cylindrically symmetric spacetimes.

From the definition of the lapse density $N$ and the time $T$ or, equivalently, from the equations of motion for $T$, it follows that the rate of change of $T(\infty)$ with respect to $t$ is the asymptotic value of the lapse density $N(\infty)$. This motivates the following construction [24]. We introduce a new degree of freedom, $\tau_{\infty}$, which is the time displayed by a clock at infinity that measures time $T$. The lapse density at infinity is expressed as

$$
N(\infty)=\dot{\tau}_{\infty}
$$

If we insist on keeping fixed the asymptotic value of the lapse density, then we also keep fixed $\dot{\tau}_{\infty}$, and nothing is changed except notation. However, we can treat $\tau_{\infty}$ as a new dynamical variable which is to be varied in the action. This is the usual logic of the parametrization process as applied to the "point at infinity." If we parametrize at infinity, the Hamiltonian form of the action (2.34) can be written as

$$
\begin{aligned}
\widetilde{S}\left[N, N^{r}, \gamma, R, \psi, \widetilde{\psi}, \pi_{\gamma}, \pi_{R}, \pi_{\psi}, \pi_{\tilde{\psi}}, \tau_{\infty}\right] \\
=\int_{t_{1}}^{t_{2}} d t \int_{0}^{\infty} d r\left(\pi_{\gamma} \dot{\gamma}+\pi_{R} \dot{R}+\pi_{\psi} \dot{\psi}+\pi_{\tilde{\psi}} \dot{\tilde{\psi}}-N \mathscr{H}\right. \\
\left.\quad-N^{r} \mathscr{H}_{r}\right)-\int_{t_{1}}^{t_{2}} d t \dot{\tau}_{\infty} \gamma(\infty) .
\end{aligned}
$$

By adding the new variable $\tau_{\infty}$ to the Hamiltonian action principle, we get additional equations, which, however, are equivalent to the original equations. In detail, by varying $\tau_{\infty}$ we obtain conservation of the $C$ energy, $\dot{\gamma}(\infty)=0$, which already followed from the other equations of motion and so does not alter the content of the field equations. The other new equation comes from varying $\gamma$. Prior to parametrizing at infinity, the variation of $\gamma$ led to one of the field equations (2.10) and (2.11), and a potential boundary equation was eliminated by the boundary term in the Hamiltonian. After parametrization, the boundary equation survives and yields Eq. (3.5), which recovers the desired definition of $\tau_{\infty}$.

To summarize, we can enlarge the phase space $\Gamma$ of cylindrically symmetric spacetimes by adding a single variable $\tau_{\infty}$. The extended phase space will be denoted $\Gamma^{\star}$. The extrema of the action functional (3.6) still define cylindrically symmetric vacuum spacetimes, but now in terms of the extended set of variables. By enlarging the phase space in this manner, we are able to define the asymptotic location of spatial curves using dynamical variables. The action $\widetilde{S}$, however, is not in Hamiltonian form because the surface term now enters as a "kinetic term" and destroys the canonical nature of the phase space coordinates $\gamma$ and $\pi_{\gamma}$. We still must find canonical coordinates and momenta on $\Gamma^{\star}$. Indeed, we must show that $\Gamma^{\star}$ is a symplectic manifold. We will take 
care of these issues, while at the same time providing the cylindrically symmetric version of the canonical transformation (3.1), in the following.

Let us define the phase space $Y$ for a field theory on $M$ as follows. A point in phase space is defined by the functions $\left(T, R, \psi, \widetilde{\psi}, \Pi_{T}, \Pi_{R}, \pi_{\psi}, \pi_{\tilde{\psi}}\right)$ on $\mathbf{R}^{+}$, where $(T, R, \psi, \widetilde{\psi})$ are scalar functions and $\left(\Pi_{T}, \Pi_{R}, \pi_{\psi}, \pi_{\tilde{\psi}}\right)$ are scalar densities of weight one. Boundary and fall off conditions on these scalar fields and scalar densities are as indicated in the Appendix. We will need some restrictions on the functions $T(r)$ and $R(r)$ so that they can be interpreted as spacelike embeddings of $\mathbf{R}^{+}$into $M$. For reasons which will be clearer in a moment, we demand

$$
R^{\prime}>\left|T^{\prime}\right|
$$

and we include this inequality in the definition of $Y$. We define a symplectic two-form, $\Xi(X, \hat{X})$ on $Y$ by its action on a pair of vectors

$$
X=\left(\delta T, \delta R, \delta \psi, \delta \widetilde{\psi}, \delta \Pi_{T}, \delta \Pi_{R}, \delta \pi_{\psi}, \delta \pi_{\psi}\right)
$$

and

$$
\hat{X}=\left(\hat{\delta} T, \hat{\delta} R, \hat{\delta} \psi, \hat{\delta} \tilde{\psi}, \hat{\delta} \Pi_{T}, \hat{\delta} \Pi_{R}, \hat{\delta} \pi_{\psi}, \hat{\delta} \pi_{\psi}\right)
$$

at a point of $Y$. The symplectic form is defined by

$$
\begin{aligned}
\Xi(X, \hat{X})= & \int_{0}^{\infty} d r\left(\delta \Pi_{T} \hat{\delta} T+\delta \Pi_{R} \hat{\delta} R+\delta \pi_{\psi} \hat{\delta} \psi+\delta \pi_{\psi} \tilde{\psi} \hat{\delta} \widetilde{\psi}\right. \\
& -[\delta \leftrightarrow \hat{\delta}]) .
\end{aligned}
$$

For the moment, the symplectic manifold $(Y, \Xi)$ is to be viewed as logically independent of the gravitational phase space; that is, $Y$ is being thought of as simply a space of functions $\left(T, R, \psi, \widetilde{\psi}, \Pi_{T}, \Pi_{R}, \pi_{\psi}, \pi_{\psi}\right)$ upon which we have defined a symplectic structure. The symplectic structure is defined so that $(T, R, \psi, \widetilde{\psi})$ and $\left(\Pi_{T}, \Pi_{R}, \pi_{\psi}, \pi_{\tilde{\psi}}\right)$ are, respectively, canonical coordinates and momenta.

Now consider the following map from $\Gamma^{\star}$ to $Y$. As suggested by our notation, we will identify the variables $R, \psi, \widetilde{\psi}, \pi_{\psi}, \pi_{\psi}$ in $\Gamma^{\star}$ and $Y$. The remaining portion of the map is defined by

$$
\begin{gathered}
T(r)=\tau_{\infty}-\int_{\infty}^{r} d \bar{r} \pi_{\gamma}(\bar{r}), \\
\Pi_{T}=-\gamma^{\prime}+\left[\ln \left(R^{\prime 2}-\pi_{\gamma}^{2}\right)\right]^{\prime}, \\
\Pi_{R}=\pi_{R}+\left[\ln \left(\frac{R^{\prime}-\pi_{\gamma}}{R^{\prime}+\pi_{\gamma}}\right)\right]^{\prime} .
\end{gathered}
$$

Note that this transformation is consistent with $\Pi_{T}$ and $\Pi_{R}$ being scalar densities of weight one. This map can be inverted; the relevant formulas are

$$
\begin{gathered}
\tau_{\infty}=T(\infty), \\
\gamma(r)=\ln \left(R^{\prime 2}-T^{\prime 2}\right)-\int_{0}^{r} d \bar{r} \Pi_{T}(\bar{r}),
\end{gathered}
$$

$$
\begin{gathered}
\pi_{\gamma}=-T^{\prime} \\
\pi_{R}=\Pi_{R}-\left[\ln \left(\frac{R^{\prime}+T^{\prime}}{R^{\prime}-T^{\prime}}\right)\right]^{\prime} .
\end{gathered}
$$

The transformation (3.11)-(3.13) is a diffeomorphism which identifies $\Gamma^{\star}$ and $Y$. In particular, the inequality (3.7) is precisely the restriction (2.24) on $\Gamma^{\star}$ and is needed for the transformation to be well defined. We can use this diffeomorphism to express the action (2.34) as a functional $S^{\star}$ of curves in $\mathrm{Y}$ :

$$
\begin{aligned}
S^{\star} & {\left[N, N^{r}, T, R, \psi, \widetilde{\psi}, \Pi_{T}, \Pi_{R}, \pi_{\psi}, \pi_{\tilde{\psi}}\right] } \\
& =\int_{t_{1}}^{t_{2}} d t \int_{0}^{\infty} d r\left(\Pi_{T} \dot{T}+\Pi_{R} \dot{R}+\pi_{\psi} \dot{\psi}+\pi_{\tilde{\psi}} \tilde{\tilde{\psi}}-N H-N^{r} H_{r}\right),
\end{aligned}
$$

where

$$
\begin{aligned}
H= & \Pi_{T^{\prime}} R^{\prime} \Pi_{R} T^{\prime}+\frac{1}{2}\left(R \psi^{\prime 2}+R^{-1} \pi_{\psi}^{2}\right) \\
& +\frac{1}{2}\left(R e^{-2 \psi} \pi_{\widetilde{\psi}}^{2}+R^{-1} e^{2 \psi} \widetilde{\psi^{\prime 2}}\right) \approx 0, \\
H_{r}= & \Pi_{T} T^{\prime}+\Pi_{R} R^{\prime}+\pi_{\psi} \psi^{\prime}+\pi_{\widetilde{\psi}} \widetilde{\psi^{\prime}} \approx 0 .
\end{aligned}
$$

Note that the surface term contribution to the action has dropped out. Indeed, from this action we see that the variables $(T, R, \psi, \widetilde{\psi})$ and $\left(\Pi_{T}, \Pi_{R}, \pi_{\psi}, \pi_{\bar{\psi}}\right)$ are canonical coordinates and momenta for the phase space $\Gamma^{\star}$. We have (i) shown that $\Gamma^{\star}$ is a symplectic manifold by exhibiting a diffeomorphism from $\Gamma^{\star}$ to the symplectic manifold $(Y, \Xi)$ and (ii) exhibited a canonical coordinate chart $\left(T, R, \psi, \widetilde{\psi}, \Pi_{T}, \Pi_{R}, \pi_{\psi}, \pi_{\psi}\right)$ on $\Gamma^{\star}$.

The action $S^{\star}$ has a nice mathematical interpretation in terms of a parametrized field theory formulation of harmonic maps on a flat on spacetime, and we shall now spend a little time developing this interpretation. Recall that harmonic maps $\varphi^{A}: \mathbf{M} \longrightarrow \mathbf{L}$ are fields on a spacetime (M,g) taking values in a Riemannian manifold $(\mathbf{L}, \sigma)$ and which extremize the " "energy integral"':

$$
I\left[\varphi^{A}\right]=-\frac{1}{2} \int_{\mathbf{M}} \sqrt{-g} g^{\alpha \beta} \sigma_{A B}(\varphi) \varphi_{, \alpha}^{A} \varphi_{, \beta}^{B},
$$

where $g_{\alpha \beta}$ is the metric on $\mathbf{M}$ and $\sigma_{A B}$ is the metric on $\mathbf{L}$. Note that we are using capital Latin indices to label the harmonic maps; in this discussion, these indices should not be confused with those labeling the Killing vectors used to define the gravitational model. Also, the spacetime manifold $\mathbf{M}$ and metric $g_{\alpha \beta}$ for the harmonic map theory should not be confused with the gravitational $\mathscr{C}$ and $g_{\alpha \beta}$.

Introduce a spacelike foliation $X: \mathbf{R} \times \Sigma \rightarrow \mathbf{M}$ characterized by lapse $N^{\perp}$ and shift $N^{i}$. The Hamiltonian form of the action (3.21) is

$$
I\left[\varphi^{A}, \pi_{A}\right]=\int_{\mathbf{R} \times \Sigma}\left(\pi_{A} \dot{\varphi}^{A}-N^{\perp} h_{\perp}-N^{i} h_{i}\right)
$$

where lowercase Latin indices denote tensors on $\Sigma$, and the energy and momentum densities are 


$$
\begin{gathered}
h_{\perp}=\frac{1}{2}\left(\frac{1}{\sqrt{q}} \sigma^{A B} \pi_{A} \pi_{B}+\sqrt{q} q^{i j} \sigma_{A B} \varphi_{, i}^{A} \varphi_{, j}^{B}\right), \\
h_{i}=\pi_{A} \varphi_{, i}^{A} .
\end{gathered}
$$

Here $q_{i j}$ is the induced metric on each hypersurface $\Sigma$ of the foliation. Variation of $I\left[\varphi^{A}, \pi_{A}\right]$ with respect to $\varphi^{A}$ and $\pi_{A}$ yields equations equivalent to those obtained by varying (3.21). At this point the spacetime metric and foliation, while arbitrary, are fixed. This is reflected by the fact that the lapse and shift are fixed fields on $\mathbf{R} \times \Sigma$, i.e., not subject to variation in the action principle. Because of this, the field theory, in either the Lagrangian or Hamiltonian formulation, is not "generally covariant." General covariance can be introduced into the field theory by keeping the spacetime metric fixed and adding the foliation itself to the space of dependent variables to be varied in the action principle. This is conveniently done in the Hamiltonian formulation, where the new dynamical variables are spacelike embeddings, which we shall denote by $X^{\alpha}$. We must still introduce momenta $\Pi_{\alpha}$ conjugate to the embeddings. To do this we need the unit normal $n^{\alpha}$ to the hypersurface embedded by $X^{\alpha}$. The unit normal is defined by

$$
g_{\alpha \beta}(X) n^{\alpha} X_{, i}^{\beta}=0 \quad \text { and } g_{\alpha \beta}(X) n^{\alpha} n^{\beta}=-1 \text {, }
$$

where $g_{\alpha \beta}(X)$ is the metric on $\mathbf{M}$ restricted to the embedding $X^{\alpha}$. The unit normal is a fixed local function of the embedding and its first spatial derivatives. The foliation is a oneparameter family of spacelike embeddings, which we shall denote by $X^{\alpha}(t)$. It is straightforward to show that

$$
\dot{X}^{\alpha}(t)=N^{\perp} n^{\alpha}+N^{i} X_{, i}^{\alpha}
$$

The action (3.22) can thus be written as

$$
I\left[\varphi^{A}, \pi_{A}\right]=\int_{\mathbf{R} \times \Sigma}\left(\pi_{A} \dot{\varphi}^{A}-\dot{X}^{\alpha} h_{\alpha}\right),
$$

where

$$
h_{\alpha}=-n_{\alpha} h_{\perp}+X_{\alpha}^{i} h_{i},
$$

and we have introduced fields $X_{\alpha}^{i}$, which are fixed local functions of the embeddings and their first spatial derivatives defined by

$$
X_{\alpha}^{i} X_{, j}^{\alpha}=\delta_{j}^{i} \quad \text { and } n^{\alpha} X_{\alpha}^{i}=0
$$

From this form of the action we see that the momenta conjugate to the embeddings are given by

$$
\Pi_{\alpha}=-h_{\alpha}
$$

these definitions represent constraints

$$
H_{\alpha}:=\Pi_{\alpha}+h_{\alpha} \approx 0
$$

We can take the constraints (3.31) into account with Lagrange multipliers $N^{\alpha}$ and obtain the final form for the Hamiltonian action describing the parametrized field theory:

$$
I\left[N^{\alpha}, X^{\alpha}, \Pi_{\alpha}, \varphi^{A}, \pi_{A}\right]=\int_{\mathbf{R} \times \Sigma}\left(\pi_{A} \dot{\varphi}^{A}+\Pi_{\alpha} \dot{X}^{\alpha}-N^{\alpha} H_{\alpha}\right) .
$$

The extrema of this action, obtained by varying it with respect to its arguments, are defined by a system of equations equivalent to those obtained by extremizing (3.27). Note that the Hamiltonian can be expressed as

$$
\mathbf{H}:=\int_{\Sigma} N^{\alpha} H_{\alpha}=\int_{\Sigma}\left(N^{\perp} H_{\perp}+N^{i} H_{i}\right)
$$

where

$$
\begin{gathered}
H_{\perp}=n^{\alpha} \Pi_{\alpha}+h_{\perp}, \\
H_{i}=X_{, i}^{\alpha} \Pi_{\alpha}+h_{i},
\end{gathered}
$$

and we can equally well vary $N^{\perp}$ and $N^{i}$ instead of $N^{\alpha}$ in (3.32).

The resulting formalism, in which the embeddings and their conjugate momenta are adjoined to the phase space [at the expense of the constraints (3.31)], is the "parametrized formalism' for the harmonic map field theory. The central feature of the parametrized formalism is that it provides a generally covariant formulation of any field theory. This is what makes possible the identification of the gravitational models, which are generally covariant field theories on $M$, with the theory of harmonic maps on a fixed background spacetime.

Now we are ready to make contact with the Hamiltonian formulation of cylindrically symmetric spacetimes. This is accomplished in three steps.

\section{Fix the spacetime and target space}

We fix the spacetime to be $\mathbf{M}=\mathbf{R}^{2} \times \mathbf{S}^{1}$ equipped with a flat metric, which is defined in polar coordinates by the line element

$$
g_{\alpha \beta} d x^{\alpha} d x^{\beta}=-d T^{2}+d R^{2}+R^{2} d \Phi^{2} .
$$

Here $T \in(-\infty, \infty), R \in(0, \infty)$, and $\Phi \in(0,2 \pi)$. The harmonic maps are defined to take values in $\mathbf{L}=\mathbf{R}^{2}$ with the metric $\sigma_{A B}$ chosen to be of constant negative curvature. In coordinates $\psi \in(-\infty, \infty)$ and $\phi \in(-\infty, \infty)$, the metric on $\mathbf{L}$ is determined by the line element

$$
\sigma_{A B} d \varphi^{A} d \varphi^{B}=d \psi^{2}+e^{-2 \psi} d \phi^{2} .
$$

The scalar curvature of this metric is -2 .

\section{Impose azimuthal symmetry}

Of course $\partial / \partial \Phi$ is a Killing vector field of the metric $g_{\alpha \beta}$. We now demand that the fields $\varphi^{A}$ be invariant along the flow generated by this Killing vector field: i.e.,

$$
\mathscr{L}_{\partial / \partial \Phi} \varphi^{A}=0 .
$$

In the coordinates $(T, R, \Phi)$ on $\mathbf{M}$, this means we assume the fields are independent of $\Phi$ :

$$
\psi=\psi(T, R) \text { and } \phi=\phi(T, R) .
$$


In the parametrized formalism for the field theory, we also assume that the foliation is compatible with the azimuthal symmetry in the sense that we only consider spacelike surfaces to which $\partial / \partial \Phi$ is everywhere tangent. The embeddings can be registered in the coordinates $(T, R, \Phi)$; that is, we have

$$
X^{\alpha}(r, \Phi)=(T(r), R(r), \Phi)
$$

where $r \in(0, \infty)$. Thus, to specify an embedding, we must specify two functions of one variable, $T(r)$ and $R(r)$. On the symmetry-compatible foliation the shift vector takes the form

$$
N^{i}=\left(N^{r}, 0\right)
$$

We can now formulate the parametrized harmonic map field theory on the two-dimensional space of orbits $M=\mathbf{R} \times \mathbf{R}^{+}$of the Killing vector. By working in polar coordinates on $\mathbf{M}$, this amounts to simply ignoring the $\Phi$ coordinate. The Hamiltonian form of the action can be obtained by substituting the choices made in the first two steps into the action (3.32) and integrating out the angular coordinate. We obtain

$$
\begin{aligned}
I\left[N^{\perp}, N^{r}, T, R, \Pi_{T}, \Pi_{R}, \psi, \phi, \pi_{\psi}, \pi_{\phi}\right] \\
=\int_{t_{1}}^{t_{2}} d t \int_{0}^{\infty} d r\left(\Pi_{T} \dot{T}+\Pi_{R} \dot{R}+\pi_{\psi} \dot{\psi}\right. \\
\left.+\pi_{\phi} \dot{\phi}-N^{\perp} H_{\perp}-N^{r} H_{r}\right),
\end{aligned}
$$

where

$$
\begin{gathered}
H_{\perp}=\frac{1}{\sqrt{R^{\prime 2}-T^{\prime 2}}}\left[\Pi_{T} R^{\prime}+\Pi_{R} T^{\prime}+\frac{1}{2}\left(R \psi^{\prime 2}+R^{-1} \pi_{\psi}^{2}\right)\right. \\
\left.+\frac{1}{2}\left(R e^{-2 \psi} \phi^{\prime 2}+R^{-1} e^{2 \psi} \pi_{\phi}^{2}\right)\right] \\
H_{r}=\Pi_{T} T^{\prime}+\Pi_{R} R^{\prime}+\pi_{\psi} \psi^{\prime}+\pi_{\phi} \phi^{\prime}
\end{gathered}
$$

Variation of this action with respect to its arguments yields field equations equivalent to those obtained from (3.32) in the special case of azimuthal symmetry. An equivalent variational principle is obtained by defining a lapse density

$$
N:=\frac{1}{\sqrt{R^{\prime 2}-T^{\prime 2}}} N^{\perp}
$$

and weight-two super-Hamiltonian

$$
\begin{aligned}
H= & \Pi_{T^{\prime}} R^{\prime}+\Pi_{R} T^{\prime}+\frac{1}{2}\left(R \psi^{\prime 2}+R^{-1} \pi_{\psi}^{2}\right) \\
& +\frac{1}{2}\left(R e^{-2 \psi} \phi^{\prime 2}+R^{-1} e^{2 \psi} \pi_{\phi}^{2}\right) .
\end{aligned}
$$

In terms of these quantities we have

$$
\begin{aligned}
\bar{I}\left[N, N^{r}, T, R, \Pi_{T}, \Pi_{R}, \psi, \phi, \pi_{\psi}, \pi_{\phi}\right] \\
=\int_{t_{1}}^{t_{2}} d t \int_{0}^{\infty} d r\left(\Pi_{T} \dot{T}+\Pi_{R} \dot{R}+\pi_{\psi} \dot{\psi}+\pi_{\phi} \dot{\phi}\right. \\
\\
\left.-\dot{N} H-N^{r} H_{r}\right),
\end{aligned}
$$

and variation of this action with respect to its arguments yields field equations equivalent to those obtained from (3.42). Note the close similarity between the superHamiltonian (3.46) for the harmonic map theory and the super-Hamiltonian (3.19) for cylindrically symmetric spacetimes.

\section{Canonical transformation}

In the last step, we perform a canonical transformation that interchanges the roles of $\phi$ and $\pi_{\phi}$ and puts the superHamiltonian and supermomentum of the parametrized field theory of harmonic maps into the form (3.19) and (3.20). This transformation is given by

$$
\begin{gathered}
\widetilde{\psi}(r)=\int_{\infty}^{r} d \bar{r} \pi_{\phi}(\bar{r}), \\
\pi_{\psi}=\phi^{\prime} .
\end{gathered}
$$

The inverse transformation is

$$
\begin{gathered}
\phi(r)=\int_{0}^{r} d \bar{r} \pi_{\tilde{\psi}}(\vec{r}), \\
\pi_{\phi}=\widetilde{\psi}^{\prime} .
\end{gathered}
$$

With the boundary conditions given in the Appendix, this is a canonical transformation provided we impose the boundary condition $\phi(r=0)=0$. It follows immediately from (3.50) and (3.51) that, in the new variables, the super-Hamiltonian and supermomentum for the parametrized harmonic map field theory are precisely (3.19) and (3.20).

As indicated by the equivalence to a parametrized field theory, the constraints (3.19) and (3.20),

$$
H \approx 0 \approx H_{r},
$$

can be solved for the momenta conjugate to the embeddings. Indeed, it is straightforward to show that these constraints are equivalent to

$$
H_{a}:=\Pi_{a}+h_{a} \approx 0
$$

where $\Pi_{a}=\left(\Pi_{T}, \Pi_{R}\right)$ and $h_{a}=\left(h_{T}, h_{R}\right)$ is defined as in (3.28) with

$$
\begin{gathered}
n_{a}=\left(n_{T}, n_{R}\right)=\left(-\frac{R^{\prime}}{\sqrt{R^{\prime 2}-T^{\prime 2}}}, \frac{T^{\prime}}{\sqrt{R^{\prime 2}-T^{\prime 2}}}\right), \\
X_{a}^{r}=\left(X_{T}^{r}, X_{R}^{r}\right)=\left(-\frac{T^{\prime}}{R^{\prime 2}-T^{\prime 2}}, \frac{R^{\prime}}{R^{\prime 2}-T^{\prime 2}}\right) .
\end{gathered}
$$

Explicitly, the constraints (3.53) take the form

$$
\begin{array}{r}
\Pi_{T}+\frac{1}{R^{\prime 2}-T^{\prime 2}}\left(R ^ { \prime } \left[\frac{1}{2}\left(R \psi^{\prime 2}+R^{-1} \pi_{\psi}^{2}\right)+\frac{1}{2}\left(R e^{-2 \psi} \pi_{\tilde{\psi}}^{2}\right.\right.\right. \\
\left.\left.\left.+R^{-1} e^{2 \psi}{\widetilde{\psi^{\prime}}}^{2}\right)\right]-T^{\prime}\left(\pi_{\psi} \psi^{\prime}+\pi_{\tilde{\psi}} \widetilde{\psi^{\prime}}\right)\right) \approx 0
\end{array}
$$

and 


$$
\begin{array}{r}
\Pi_{R}-\frac{1}{R^{\prime 2}-T^{\prime 2}}\left(T ^ { \prime } \left[\frac{1}{2}\left(R \psi^{\prime 2}+R^{-1} \pi_{\psi}^{2}\right)+\frac{1}{2}\left(R e^{-2 \psi} \pi_{\widetilde{\psi}}^{2}\right.\right.\right. \\
\left.\left.\left.+R^{-1} e^{2 \psi}{\widetilde{\psi^{\prime}}}^{2}\right)\right]-R^{\prime}\left(\pi_{\psi} \psi^{\prime}+\pi_{\widetilde{\psi}} \widetilde{\psi}^{\prime}\right)\right) \approx 0 .
\end{array}
$$

A standard argument (see, e.g., [25]) establishes that the constraints (3.56) and (3.57) have an Abelian Poisson bracket algebra:

$$
\left\{H_{a}(r), H_{b}(\vec{r})\right\}=0 .
$$

To summarize, the Hamiltonian structure of cylindrically symmetric gravitational fields is mathematically identical to a parametrized field theory of azimuthally symmetric harmonic maps from a three-dimensional flat spacetime to a two-dimensional manifold equipped with a metric of constant negative curvature. It is important to note that all of these results are fully gauge invariant in the sense that no coordinate conditions are needed to be imposed on the gravitational theory. In any of the forms that we have presented the Hamiltonian formulation, the field theory retains the full two-dimensional diffeomorphism invariance compatible with the imposition of cylindrical symmetry.

\section{B. Closed universes: Toroidal symmetry}

We now repeat the analysis of Sec. III A under the assumption of toroidal symmetry. The procedure is very similar to that used in the cylindrical symmetry case; the key difference is the way in which the missing degree of freedom is introduced.

Again, the strategy is to use the conformal coordinates $\tau$ and $X$ to define embeddings of a circle into $M=\mathbf{R}^{+} \times \mathbf{S}^{1}$. The variable $\tau$ already appears as a canonical coordinate on the phase space, but we must still express $X$ as a function on phase space. This can be achieved starting from (2.39). Choosing the spacelike circle on which the integral is performed to be a $t=$ const slice, we have that

$$
X^{\prime}=\frac{1}{\pi_{0}} \pi_{\gamma},
$$

where

$$
\pi_{0}:=\frac{1}{2 \pi} \int_{0}^{2 \pi} d x \pi_{\gamma}(x)
$$

Note that, because of (2.53), $\pi_{\gamma}$ is negative definite, and so the denominator never vanishes in (3.59) and $X(x)$ is monotonic.

Before completing the phase space definition of $\tau$ and $X$ into a canonical transformation, we have to contend with the fact that (3.59) does not define $X$ as a function on the gravitational phase space $\Gamma$. The reason is the same as in the cylindrically symmetric case: There is an integration constant left unspecified in (3.59), which is not fixed by the gravitational phase space data. In Sec. III A the integration constant represented the asymptotic location of a spacelike slice; here, it represents the relation between the origin of the coordinate $x$ on $\mathbf{S}^{1}$ and the origin of the conformal coordinate $X$. This information is coordinate dependent and not included in the gravitational phase space. As before, we rem- edy this situation by adding a new degree of freedom $q$ to the phase space [24]. Unlike the cylindrically symmetric case, this new degree of freedom has no role to play in gravitational dynamics; i.e., it is pure gauge. We therefore introduce a momentum $p$ conjugate to $q$ and adjoin a new constraint,

$$
p \approx 0,
$$

to the Hamiltonian formulation. We denote by $\Gamma^{\star}$ the phase space extended by the variables $q$ and $p$. Our strategy is to formulate the gravitational system as a parametrized field theory on $\Gamma^{\star}$ and then reduce the system by the constraint (3.61). When we reduce $\Gamma^{\star}$ by the constraint (3.61), we arrive at the original gravitational phase space $\Gamma$ and the dynamics thereon.

We are now ready to extract embedding variables from the gravitational system formulated on $\Gamma^{\star}$. Let $\mu(x)$ be a prescribed measure on the circle; i.e., $\mu$ is a positive density of weight one on the circle and is normalized via

$$
\int_{0}^{2 \pi} d x \mu(x)=1
$$

We define a transformation

$\left(\tau, \gamma, \psi, \widetilde{\psi}, q, \pi_{\tau}, \pi_{\gamma}, \pi_{\psi}, \pi_{\tilde{\psi}}, p\right)$

$$
\leftrightarrow\left(T, X, \phi, \widetilde{\phi}, \mathscr{Q}, \Pi_{T}, \Pi_{X}, \pi_{\phi}, \pi_{\tilde{\phi}}, \mathscr{P}\right)
$$

by

$$
\begin{gathered}
T=-\frac{1}{\pi_{0}} \tau \\
X(x)=q+\int_{0}^{2 \pi} d x^{\prime \prime} \mu\left(x^{\prime \prime}\right) \int_{x^{\prime \prime}}^{x} d x^{\prime} \frac{1}{\pi_{0}} \pi_{\gamma}\left(x^{\prime}\right),
\end{gathered}
$$

$$
\begin{gathered}
\Pi_{T}=-\pi_{0}\left(\pi_{\tau}+\left[\ln \left(\frac{\pi_{\gamma}-\tau^{\prime}}{\tau^{\prime}+\pi_{\gamma}}\right)\right]^{\prime}\right), \\
\Pi_{X}=p \mu+\pi_{0}\left(\gamma^{\prime}-\left[\ln \left(\pi_{\gamma}^{2}-\tau^{\prime 2}\right)\right]^{\prime}\right)
\end{gathered}
$$

and

$$
\begin{gathered}
\phi=\sqrt{-\pi_{0}} \psi, \\
\widetilde{\phi}=\frac{1}{\sqrt{-\pi_{0}}} \widetilde{\psi}, \\
\pi_{\phi}=\frac{1}{\sqrt{-\pi_{0}}} \pi_{\psi}, \\
\pi_{\tilde{\phi}}=\sqrt{-\pi_{0}} \pi_{\tilde{\psi}},
\end{gathered}
$$




$$
\begin{gathered}
Q=\frac{1}{\pi_{0}} \int_{0}^{2 \pi} d x\left\{\left[\gamma-\ln \left(\pi_{\gamma}^{2}-\tau^{\prime 2}\right)\right] \pi_{\gamma}\right. \\
-\left(\pi_{\tau}+\left[\ln \left(\frac{\pi_{\gamma}-\tau^{\prime}}{\tau^{\prime}+\pi_{\gamma}}\right)\right]^{\prime}\right) \tau \\
\left.+\frac{1}{2} \pi_{\psi} \psi-\frac{1}{2} \pi_{\bar{\psi}} \widetilde{\psi}\right\}, \\
\mathscr{P}=\pi_{0} .
\end{gathered}
$$

Here we have made some convenient rescalings. In particular, the scaling by $1 / \pi_{0}$ used in the definition of $X$ guarantees that

$$
X(2 \pi)-X(0)=2 \pi
$$

We mention that the requirement that $D_{a} \tau$ be timelike, given by $(2.53)$, is equivalent to

$$
X^{\prime}>\left|T^{\prime}\right|
$$

The inequalities (2.53) and (3.75) guarantee that the above transformation is well defined and that the slices embedded by $(T(x), X(x))$ are spacelike. Let us also note that the constraint (3.61) is, in the new variables, the constraint

$$
\int_{0}^{2 \pi} d x \Pi_{X}(x) \approx 0
$$

It is not too hard to show, e.g., by expanding in Fourier series, that the above transformation is a bijection from $\Gamma^{\star}$ to the phase space $Y$ for a field theory of $\left(\phi, \widetilde{\phi}, \mathcal{Q}, \pi_{\phi}, \pi_{\bar{\phi}}, \mathscr{P}\right)$ in the parametrized formalism. Our notation here is that $Y$ is the product of the cotangent bundle over the space of embeddings of a circle into $M$ and the phase space of the canonical variables $(\phi, \widetilde{\phi}, Q)$ and $\left(\pi_{\phi}, \pi_{\tilde{\phi}}, \mathscr{P}\right)$. By computing Poisson brackets, or by computing the symplectic form in the new variables, it is straightforward to verify that the transformation is canonical, i.e., identifies the respective symplectic structures, and that the variables $(T, X, \phi, \widetilde{\phi}, \mathcal{Q})$ and $\left(\Pi_{T}, \Pi_{X}, \pi_{\phi}, \pi_{\tilde{\phi}}, \mathscr{P}\right)$ are canonical coordinates and momenta.

Modulo the constraint (3.76), the weight-two superHamiltonian and supermomentum take the following form when expressed in terms of the new canonical variables:

$$
\begin{aligned}
H:= & \Pi_{T} X^{\prime}+\Pi_{X} T^{\prime}+\frac{1}{2}\left(T \phi^{\prime 2}+T^{-1} \pi_{\phi}^{2}\right) \\
& +\frac{1}{2}\left(T e^{-2 \phi / \sqrt{-\mathscr{P}}} \pi_{\widetilde{\phi}}^{2}+T^{-1} e^{2 \phi / \sqrt{-\mathscr{P}}} \widetilde{\phi}^{\prime 2}\right) \approx 0
\end{aligned}
$$

and

$$
H_{x}:=\Pi_{T} T^{\prime}+\Pi_{X} X^{\prime}+\pi_{\phi} \phi^{\prime}+\pi_{\bar{\phi}} \widetilde{\phi}^{\prime} \approx 0 .
$$

The constraints of the theory are (3.76)-(3.78); they are "'first class.' In particular, the Poisson brackets of the constraint (3.76) with the super-Hamiltonian and supermomen- tum vanish because (3.76) generates constant shifts of $X$ with respect to which $H$ and $H_{x}$ are invariant.

Let us make one further remark about the canonical transformation (3.63). First, we emphasize that the identification of $\Gamma^{\star}$ with $Y$ depends on the nongravitational data $(q, p, \mu)$. However, upon passing to $\Gamma$ using the constraint (3.76), this dependence necessarily disappears. To see this, recall that the reduction from $\Gamma^{\star}$ to $\Gamma$ is obtained by (i) restricting the phase space to the constraint surface defined by (3.76) and (ii) identifying points on the constraint surface which lie on an orbit of the canonical transformations generated by $\int_{s^{1}} \Pi_{X}$; that is, embeddings $X$ and $X+$ const are identified. The dependence of $\Pi_{X}$ on $p$ and $\mu$ is eliminated upon passing to the constraint surface defined by (3.76). Restricting to the constraint surface of (3.76), we must identify $X$ and $X+$ const. For a fixed choice of $\mu$ this eliminates the dependence of the phase space on $q$. Now suppose we use a different measure $\tilde{\mu}$ to define a new embedding variable $\widetilde{X}(x)$. Because $\widetilde{X}^{\prime}=\pi_{\gamma} / \pi_{0}$, we have that $(\widetilde{X}-X)^{\prime}=0$, that is, $\widetilde{X}=X$ + const, which is precisely the transformation generated by $\int_{\mathbf{s}^{1}} \Pi_{X}$, and so on $\Gamma$ the phase space is independent of the choice of measure $\mu$. Put differently, changing $\mu$ is equivalent to holding $\mu$ fixed and changing $q$, which has no effect on points of $\Gamma$. Thus the nongravitational data $(q, p, \mu)$ are eliminated upon passing from $\Gamma^{\star}$ to the original phase space $\Gamma$.

Once again we can interpret the resulting formalism in terms of a parametrized field theory of harmonic maps. However, it must be kept in mind that there is a "point particle", degree of freedom represented by $\mathcal{Q}$ and $\mathscr{P}$ along with an extra constraint (3.76). Because $Q$ is cyclic in the Hamiltonian, we can reduce the phase space $\Gamma^{\star}$ by the integral of motion $\mathscr{P}$ to a phase space $\widetilde{\Gamma}^{\star}$. On $\widetilde{\Gamma}^{\star}$ we view $\mathscr{P}$ as a parameter, which will appear in the metric for the spacetime upon which the harmonic maps are defined. To express the gravitational theory formulated on $\widetilde{\Gamma}^{\star}$ as a parametrized harmonic map field theory, we repeat the three steps of Sec. III A.

\section{Fix the spacetime and target space}

The spacetime is now taken to be $\mathbf{M}=\mathbf{R}^{+} \times \mathbf{T}^{2}$ with metric given by the line element

$$
d s^{2}=l^{2}\left(-d T^{2}+d X^{2}+T^{2} d Y^{2}\right)
$$

where $X$ and $Y$ are coordinates on $\mathbf{T}^{2}, T>0$, and $l$ is a positive constant. Using an analogous construction to that found in Sec. II B, this metric can be viewed as a flat metric on a compactification of a submanifold $I$ of three-dimensional Minkowski spacetime $\mathbf{M}^{3}$. Let us spell this out in detail. Let $\hat{t}, \hat{x}, \hat{y}$ denote inertial coordinates on $\mathbf{M}^{3}$. Define $I$ as the submanifold for which $\hat{t}^{2}-\hat{y}^{2}>0$ and $\hat{t}>0$. On $I$ make the identification

$$
\hat{x} \sim \hat{x}+2 \pi l, \quad \operatorname{arctanh}\left(\frac{\hat{y}}{\hat{t}}\right) \sim \operatorname{arctanh}\left(\frac{\hat{y}}{\hat{t}}\right)+2 \pi
$$

and denote the resulting manifold by $\widetilde{I}$. The Minkowski metric on $\mathbf{M}^{3}$ projects to a flat metric on $\widetilde{I}$. Define coordinates $(T, X, Y)$ on $\mathbf{R}^{+} \times \mathbf{T}^{2}$, where $T \in(0, \infty), X \in(-\infty, \infty)$, and $Y$ 
$\in(-\infty, \infty)$, with $X \sim X+2 \pi$ and $Y \sim Y+2 \pi$. The diffeomorphism $\phi: \mathbf{R}^{+} \times \mathbf{T}^{2} \rightarrow \widetilde{I}$, defined by

$$
\begin{gathered}
\hat{t}=l T \cosh Y, \\
\hat{x}=l X, \\
\hat{y}=l T \sinh Y,
\end{gathered}
$$

identifies the metric (3.79) on $\mathbf{R}^{+} \times \mathbf{T}^{2}$ with the Minkowski metric on $\widetilde{I}$.

As in the cylindrically symmetric case, the harmonic maps $\varphi^{A}:=(\alpha, \beta)$ take values in $\mathbf{L}=\mathbf{R}^{2}$, which is equipped with a metric of constant negative curvature given by the line element

$$
\sigma_{A B} d \varphi^{A} d \varphi^{B}=d \alpha^{2}+e^{-2 \alpha} d \beta^{2} .
$$

\section{Impose azimuthal symmetry}

The vector field $\partial / \partial Y$ is a Killing vector field for the metric on $\mathbf{M}$. We now demand that all the fields of the parametrized harmonic map field theory are likewise invariant under the one-parameter family of isometries generated by $\partial / \partial Y$. Thus we can formulate the theory on the space of orbits, $M=\mathbf{R}^{+} \times \mathbf{S}^{1}$, of the Killing vector field. On the space of orbits of $\partial / \partial Y$, the Hamiltonian form of the action is given by

$$
\begin{aligned}
& \bar{I}\left[N, N^{x}, T, X, \pi_{T}, \pi_{X}, \alpha, \beta, \pi_{\alpha}, \pi_{\beta}\right] \\
&=\int_{t_{1}}^{t_{2}} d t \int_{0}^{2 \pi} d x\left(\pi_{T} \dot{T}+\pi_{X} \dot{X}+\pi_{\alpha} \dot{\alpha}+\pi_{\beta} \dot{\beta}\right. \\
&\left.-N H-N^{x} H_{x}\right),
\end{aligned}
$$

where

$$
\begin{aligned}
H= & \pi_{T} X^{\prime}+\pi_{X} T^{\prime}+\frac{1}{2}\left[(l T)^{-1} \pi_{\alpha}^{2}+l T \alpha^{\prime 2}\right] \\
& +\frac{1}{2}\left[(l T)^{-1} e^{2 \alpha} \pi_{\beta}^{2}+l T e^{-2 \alpha} \beta^{\prime 2}\right]
\end{aligned}
$$

and

$$
H_{x}=\pi_{T} T^{\prime}+\pi_{X} X^{\prime}+\pi_{\alpha} \alpha^{\prime}+\pi_{\beta} \beta^{\prime} .
$$

Note the similarity between the super-Hamiltonian (3.84) for the harmonic map theory and the super-Hamiltonian (3.77) for toroidally symmetric spacetimes.

\section{Canonical transformation}

In this last step we make a canonical transformation which puts the super-Hamiltonian and supermomentum (3.84) and (3.85) of the parametrized field theory into the form (3.77) and (3.78) found in the gravitational theory. It is possible to adapt the transformation (3.48)-(3.49) used for the cylindrically symmetric case, but this leads to additional, and unnecessary, constraints on the harmonic maps. In the toroidal symmetry case the following canonical transformation accomplishes our goal [26]:

$$
\begin{gathered}
\alpha=-l^{-1 / 2} \phi+\ln (l T), \\
\beta=l^{1 / 2} \widetilde{\phi},
\end{gathered}
$$

$$
\begin{gathered}
\pi_{\alpha}=-l^{1 / 2} \pi_{\phi}+l X^{\prime}, \\
\pi_{\beta}=l^{-1 / 2} \pi_{\bar{\phi}}, \\
\pi_{T}=\Pi_{T}+l^{1 / 2} T^{-1} \pi_{\phi}-l(2 T)^{-1} X^{\prime}, \\
\pi_{X}=\Pi_{X}+l^{1 / 2} \phi^{\prime}-l(2 T)^{-1} T^{\prime} .
\end{gathered}
$$

Note that the embedding coordinates $(T, X)$ retain their original meaning and the embedding momenta, while redefined, are still scalar densities of weight one on the circle. By using the transformation (3.86)-(3.91), it follows that the superHamiltonian and supermomentum (3.84) and (3.85) of the parametrized field theory become those found in the gravitational theory formulated on $\widetilde{\Gamma}^{\star}[(3.77)$ and (3.78)], provided we make the identification

$$
l=-\mathscr{P} .
$$

As in the cylindrically symmetric case, we now know that the constraints (3.77) and (3.78),

$$
H \approx 0 \approx H_{x},
$$

can be reexpressed as

$$
H_{a}:=\Pi_{a}+h_{a} \approx 0,
$$

where $\Pi_{a}=\left(\Pi_{T}, \Pi_{X}\right)$ and $h_{a}=\left(h_{T}, h_{X}\right)$ is defined as in (3.28) with

$$
\begin{gathered}
n_{a}=\left(n_{T}, n_{X}\right)=\left(-\frac{X^{\prime}}{\sqrt{X^{\prime 2}-T^{\prime 2}}}, \frac{T^{\prime}}{\sqrt{X^{\prime 2}-T^{\prime 2}}}\right), \\
X_{a}^{x}=\left(X_{T}^{x}, X_{X}^{x}\right)=\left(-\frac{T^{\prime}}{X^{\prime 2}-T^{\prime 2}}, \frac{X^{\prime}}{X^{\prime 2}-T^{\prime 2}}\right) .
\end{gathered}
$$

Explicitly, the constraints (3.94) take the form

$$
\begin{gathered}
\Pi_{T}+\frac{1}{X^{\prime 2}-T^{\prime 2}}\left(X ^ { \prime } \left[\frac{1}{2}\left(T \phi^{\prime 2}+T^{-1} \pi_{\phi}^{2}\right)+\frac{1}{2}\left(T e^{-2 \phi / \sqrt{-\mathscr{P}}} \pi_{\widetilde{\phi}}^{2}\right.\right.\right. \\
\left.\left.\left.+T^{-1} e^{2 \phi / \sqrt{-\mathscr{P}}} \widetilde{\phi}^{\prime 2}\right)\right]-T^{\prime}\left(\pi_{\phi} \phi^{\prime}+\pi_{\tilde{\phi}} \widetilde{\phi}^{\prime}\right)\right) \approx 0
\end{gathered}
$$

and

$$
\begin{gathered}
\Pi_{X}-\frac{1}{X^{\prime 2}-T^{\prime 2}}\left(T ^ { \prime } \left[\frac{1}{2}\left(T \phi^{\prime 2}+T^{-1} \pi_{\phi}^{2}\right)+\frac{1}{2}\left(T e^{-2 \phi / \sqrt{-\mathscr{P}}} \pi_{\widetilde{\phi}}^{2}\right.\right.\right. \\
\left.\left.\left.+T^{-1} e^{2 \phi / \sqrt{-\mathscr{P}}} \widetilde{\phi}^{\prime 2}\right)\right]-X^{\prime}\left(\pi_{\phi} \phi^{\prime}+\pi_{\widetilde{\phi}^{\prime}} \widetilde{\phi}^{\prime}\right)\right) \approx 0 .
\end{gathered}
$$

As before, the constraints (3.97) and (3.98) have an Abelian Poisson bracket algebra:

$$
\left\{H_{a}(x), H_{b}(\vec{x})\right\}=0 .
$$

To summarize, the Hamiltonian formulation on $\widetilde{\Gamma}^{\star}$ of toroidally symmetric gravitational fields is equivalent to that of a parametrized field theory of harmonic maps from a flat three-dimensional spacetime to a two-dimensional manifold of constant negative curvature. Of course, to recover the original gravitational field theory on $\Gamma$ the degrees of free- 
dom $(\mathcal{Q}, \mathscr{P})$ must be reinstated, but more importantly, the constraint (3.76) must be imposed on the harmonic map field theory. This constraint sets to zero the homogeneous mode of the embedding momentum $\Pi_{X}$ and, by virtue of (3.98), is equivalent to the constraint

$$
\int_{0}^{2 \pi} d x h_{X}(x) \approx 0
$$

This constraint can be viewed as setting to zero the total momentum of the fields $\phi$ and $\widetilde{\phi}$ (or $\alpha$ and $\beta$ ). Because the constraint (3.100) is first class, we are obliged to identify $X$ and $X+$ const. The equivalence class of embeddings thus obtained is independent of the value of the nongravitational variables $(q, p, \mu)$. On the other hand, the extra constraint means that, strictly speaking, the Hamiltonian formulation of toroidally symmetric spacetimes is not quite identical to a parametrized field theory of harmonic maps. However, as we shall see, the correspondence is sufficiently close to allow a formal construction of the quantum theory.

\section{IMPLICATIONS FOR QUANTUM THEORY}

Issues which arise in canonical quantum gravity, the problems of time in particular [1], can be investigated in the models we have been studying. In the last section we exhibited canonical transformations that identify certain phase spaces for these models with phase spaces for harmonic maps from flat three-dimensional spacetimes into a target space of constant negative curvature. This mathematical identification is fully gauge invariant, i.e., preserves the twodimensional diffeomorphism symmetry exhibited by the two Killing vector models. It should be emphasized, however, that the extraction of the embedding variables was not without its complications. We encountered a "global problem of time,' which was resolved by adding a finite number of nongravitational degrees of freedom to the usual ADM phase space. Given the extended phase spaces, each event in the effective two-dimensional spacetime is uniquely labeled by the values of the canonical variables $X^{a}$ on a spacelike slice at the point where the spacelike slice passes through that event. The identification of spacetime points provided by $X^{a}$ is independent of the choice of slice and the "spacetime problem" [1] is avoided. Given these results, the obvious strategy for quantization is to view the dynamics of the models as a generally covariant (i.e., parametrized) formulation of nonlinear fields on a fixed background. The quantum theory of fields on a fixed spacetime is quite well studied and, at least superficially, presents no overwhelming conceptual difficulties, although the quantum theory of interacting fields is always technically challenging. A simplifying feature of these models is that, while they can be viewed as field theories on a three-dimensional flat spacetime, the Killing vector structure is such that the final result is in each case a two-dimensional field theory and in two dimensions quantum field theory is typically more manageable than in higher dimensions. Our purpose in this section is to discuss certain broad features of the quantization of these models based upon the classical structures elucidated in Sec. III. We hope to return to a more detailed examination of the resulting quantum theories in future work.
In each of the models we have studied, the resulting Hamiltonian structure involves embedding variables $X^{a}$ and their conjugate momenta $\Pi_{a}$, along with dynamical variables $\left(q^{\mathbf{A}}, p_{\mathbf{A}}\right)$, which are $\left(\psi, \widetilde{\psi}, \pi_{\psi}, \pi_{\psi}\right)$ in the cylindrical symmetry case or $\left(\mathcal{Q}, \phi, \widetilde{\phi}, \mathscr{P}, \pi_{\phi}, \pi_{\tilde{\phi}}\right)$ in the toroidal symmetry case. The constraints include diffeomorphism constraints (3.53), (3.94) and, in the case of toroidal symmetry, an additional constraint (3.76). Ignoring its origins, the parametrized field theory defined by these constraints is, at least formally, relatively straightforward to "quantize." Note, however, that in each of the models the effective twodimensional field theory has a complicating feature not usually found in more familiar two-dimensional field theories. Namely, the super-Hamiltonian is an explicit function of $R(r)$ in the cylindrical symmetry case and $T(x)$ in the toroidal symmetry case. This will modify the quantum theories relative to what we might find, e.g., for a conformal field theory. In any case, we promote the canonical variables $\left(q^{\mathbf{A}}, p_{\mathbf{A}}\right)$ to operators on states $|\Psi\rangle$. One way to do this would be, using the Heisenberg picture, to quantize the variables $\left(q^{\mathbf{A}}, p_{\mathbf{A}}\right)$ on the privileged foliation $T=t$ and $R=r$ or $X=x$. The states are embedding independent in the Heisenberg picture, while the operators are evolved by the embedding momenta [27]. Of course, it may be necessary to use perturbation theory to define the operators and states. Formally, observables are self-adjoint operators representing classical functionals of $\left(q^{\mathbf{A}}, p_{\mathbf{A}}\right)$. Note that in the toroidal symmetry model observables must commute with the constraint (3.76).

In the Schrödinger picture, dynamical evolution corresponds to considering states $\left|\Psi, X^{a}\right\rangle$ which are parametrized by the embeddings. The states are evolved from one embedding to the next by the energy-momentum current $h_{a}$. More precisely, the states $\left|\Psi, X^{a}\right\rangle$ are defined as solutions to the functional Schrödinger equation

$$
i \frac{\delta}{\delta X^{a}}\left|\Psi, X^{a}\right\rangle=h_{a}\left|\Psi, X^{a}\right\rangle .
$$

This equation can be considered an implementation of the demand that "physical states are annihilated by constraints": $H_{a}\left|\Psi, X^{a}\right\rangle=0$. In the toroidal symmetry case we must also impose the quantum version of (3.76):

$$
\left(\int_{0}^{2 \pi} d x \frac{\delta}{\delta X(x)}\right)\left|\Psi, X^{a}\right\rangle=0 .
$$

This requirement is equivalent to

$$
\mid \Psi, T, X+\text { const }\rangle=|\Psi, T, X\rangle,
$$

but in light of (4.1) is also equivalent to

$$
\left(\int_{0}^{2 \pi} d x h_{X}(x)\right)\left|\Psi, X^{a}\right\rangle=0 .
$$

Given an initial state $\left|\Psi_{0}, X_{0}^{a}\right\rangle$ on an initial embedding $X_{0}^{a}$, the functional Schrödinger equation (4.1) is solved [subject to the subsidiary condition (4.2)] and the solution is matched to the initial data. Note that (4.2) can be implemented by imposing (4.3) on the initial state $\left|\Psi_{0}, X_{0}^{a}\right\rangle$ and then solving (4.1). If (4.2) is satisfied initially, it will be satisfied on any embedding provided the state is evolved according to (4.1). 
The resulting embedding-dependent vector $\left|\Psi, X^{a}\right\rangle$ is to be interpreted as the state of the system on the embedding $X^{a}(x)$, and one can go on to predict outcomes of measurements of observables constructed from the quantum fields [and $X^{a}(x)$ if desired] on various hypersurfaces in the usual way.

For this quantization strategy to be feasible, one must check that the operator representatives of the currents $h_{a}$ are well-defined operator-valued distributions. Furthermore, one must guarantee that the infinite number of differential equations contained in (4.1) and (4.2) are mutually consistent. The integrability conditions for this are the quantum commutator analogs of the Abelian algebra (3.58) and (3.99), which guarantee that quantum dynamics does not depend on the choice of observer. More precisely, the integrability conditions for (4.1) imply that the state $\left|\Psi_{1}, X_{1}^{a}\right\rangle$ obtained by evolving an initial state $\left|\Psi_{0}, X_{0}^{a}\right\rangle$ along a specific foliation connecting the slices defined by $X_{0}^{a}$ and $X_{1}^{a}$ does not depend on the choice of the foliation. For these integrability conditions to be satisfied, it is essential that the commutators of the components of the energy-momentum current be, up to a factor of $i$, the same as their classical Poisson brackets. It is very likely that this will not happen; i.e., anomalies (or "Schwinger terms") terms will arise. This is the "functional evolution problem"' [1]. It would be very interesting to compute these Schwinger terms. If they depend only on the embedding and are finite, then one can use techniques developed by Kuchar for free fields in two dimensions, where the anomalous terms are finite and depend only on the embeddings [27]. If the anomalous terms are operator valued or infinite, it is not at all clear how to proceed. Note that if we turn off one of the polarizations of the gravitational field by setting $\widetilde{\psi}=0$, then the resulting field theory is linear. In this case it would seem that all operators can be defined by normal ordering and that the Schwinger terms are finite and depend only on the embeddings, but to our knowledge no one has computed the Schwinger terms for this interesting special case. In many ways, the functional evolution problem is the most important issue to address in studying the quantization of the two Killing vector models, for it is here that one verifies the compatibility of the method of quantization with the principle of general covariance.

Given a successful resolution of the functional evolution problem, we should relate the elements of the quantum parametrized theory with geometrical elements of spacetime. In particular, what does it mean to "know the state on the embedding $X^{a}(x)$ ', ? Let us treat each of the models in turn. To fix the state $\left|\Psi, X^{a}\right\rangle$ of a cylindrically symmetric spacetime, we imagine a family of observers and the associated spacelike foliation of spacetime. On a given slice of the foliation the observers measure the gravitational variables $\left(R, \pi_{\gamma}\right)$ and the "laboratory variable" $\tau_{\infty}$; this fixes the embedding of the slice, $X^{a}(r)=(T(r), R(r))$, via (3.11). The observers also measure a complete set of commuting observables built from the operator representatives of $\left(\psi, \widetilde{\psi}, \pi_{\psi}, \pi_{\tilde{\psi}}\right)$. All together, these measurements fix the quantum state on a spacelike hypersurface in terms of measurement of geometrical quantities and the reading of a clock at infinity. In the toroidal symmetry case the family of observers measure $\tau / \pi_{0}$ and $\pi_{\gamma} / \pi_{0}$; this determines the embedding $X^{a}(x)=(T(x), X(x))$ via (3.64) and (3.65) up to specification of the nongravitational variables $q$ and $\mu$. The observers also measure a complete set of commuting observables built from $\left(\mathcal{Q}, \phi, \widetilde{\phi}, \mathscr{P}, \pi_{\phi}, \pi_{\bar{\phi}}\right)$, which are defined in terms of the spacetime geometry in (3.68)-(3.73). Different choices of $q$ and $\mu$ simply redefine $X(x)$ by addition of a constant. Because the states of a toroidally symmetric spacetime must be invariant under this transformation [see (4.3)], the quantum state $\left|\Psi, X^{a}\right\rangle$ is unambiguously determined by measurements of gravitational data only.

There is one remaining problem that should be discussed when quantizing general relativity as a parametrized field theory. Kuchar calls this problem the "multiple choice problem" [1]. The quantization we have outlined apparently depends quite heavily on the way in which the canonical variables $X^{a}$ are used to identify points of space and instants of time. To be sure, the embeddings we have constructed are, in some sense, geometrically natural. But it is conceivable that other, equally valid, embedding variables could be constructed. In this case it is not at all clear that when using some other embedding variables the resulting quantum theory will coincide with the one we have outlined here. If the quantum theories based on different choices of embedding variables do not coincide, i.e., are not physically equivalent, then we have an "embarassment of riches": Which quantum theory describes the real world? This issue can be examined in the context of the parametrized formalism for a relativistic particle moving in a curved spacetime, and it is found that the multiple choice problem can be quite severe [1]. In many ways it is the multiple choice problem which most deeply reflects the conflict between general relativity and quantum mechanics. In order to examine this problem in the models we have considered, new sets of embedding variables are required. For example, one relatively simple way to obtain other embedding variables is to take the embeddings $X^{a}$ we have constructed here and perform the point transformation

$$
\widetilde{X}^{a}:=F^{a}(X)
$$

Assuming $F^{a}$ is smooth and admits a smooth inverse, this transformation can be easily completed to a canonical transformation. These new embeddings can be interpreted as follows. Fix an embedding $X^{a}$ of a hypersurface $\Sigma$ in spacetime. Displace $\Sigma$ by letting the diffeomorphism $F^{a}$ act on it pointwise. The resulting hypersurface is embedded by $\widetilde{X}^{a}$. Is it possible to construct a quantum theory of the parametrized field theories representing the classical two Killing vector models so that transformations such as (4.5) lead to physically equivalent theories? Of course, we cannot answer this question here since we have only outlined the most basic features to be expected in the putative quantum theory. Let us, however, suggest that the multiple choice problem is closely allied with a familiar issue that arises in quantization of a gauge theory using gauge-fixing conditions. There, the question is whether or not the predictions of quantum field theories based on different gauge-fixing conditions agree. The connection of this issue with the multiple choice problem stems from the fact that, at least in the models considered here, the classical gravitational theory can be obtained by (i) choosing the gauge $T=t$ and $R=r$ or $X=x$ and (ii) "parametrizing", the resulting field theory to regain general 
covariance. Likewise, we quantize the fields $\left(q^{\mathbf{A}}, p_{\mathbf{A}}\right)$ on the foliation $T=t$ and $R=r$ or $X=x$ and then reinstate general covariance by constructing states parametrized by the embeddings $X^{a}$ which satisfy (4.1). Whether general covariance is in fact realized in the quantum theory is determined by the outcome of the problem of functional evolution. Let us assume that this problem can be solved. The remaining question is how the quantum theory depends on the initial choice of gauge. In gauge theories, this issue is fruitfully analyzed using Becchi-Rouet-Stora-Tyutin (BRST) methods [28], which, at least formally, guarantee the independence of the predictions of the quantum theory from the underlying choice of gauge. It remains to be seen whether the multiple choice problem in generally covariant theories can be resolved in a similar way.

\section{ACKNOWLEDGMENTS}

The authors would like to thank Karel Kuchar and Madhavan Varadarajan for many helpful discussions. J.D.R. was supported by Grant No. NSF-PHY-92-07225 and by research funds of the University of Utah.

\section{APPENDIX: BOUNDARY CONDITIONS FOR CYLINDRICALLY SYMMETRIC SPACETIMES}

In this appendix we summarize the boundary conditions used in the analysis of cylindrically symmetric spacetimes. These boundary conditions guarantee existence and differentiability of the various action and Hamiltonian functionals that are used in the paper, as well as the canonical nature of the various transformations considered. The boundary conditions are also such that they are preserved under the dynamical evolution generated by the Hamiltonian functionals. It is conceivable that weaker boundary conditions can be used, but those used here allow for a reasonably large class of solutions to the field equations. In particular, the boundary conditions include the Einstein-Rosen wave solutions which arise when one assumes whole cylindrical symmetry.

It is important to note that the asymptotic values $(r \rightarrow \infty)$ of the lapse and lapse density, while arbitrary, are held fixed when varying the Hamiltonian (or action) defined on the ADM phase space $\Gamma$. The asymptotic values of the lapse and lapse density are only allowed to vary when using the extended phase space $\Gamma^{\star}$ for cylindrically symmetric spacetimes. Note also that many other variables have a nonvanishing value as $r \rightarrow 0$ or $r \rightarrow \infty$; these limiting values are not held fixed in any of the variational principles. In particular, the values of the lapse and lapse density on the axis $r=0$ are not held fixed.

The phase space variables are functions of the radial coordinate $r$; we must give the boundary conditions as $r \rightarrow 0$ and $r \rightarrow \infty$. As $r \rightarrow 0$, we assume the following behavior of the canonical coordinates and momenta on $\Gamma$ :

$$
\begin{gathered}
R=r+O\left(r^{3}\right), \\
\gamma=O\left(r^{2}\right), \\
\psi=\psi(0)+O\left(r^{2}\right), \\
\widetilde{\psi}=\widetilde{\psi}(0)+O\left(r^{3}\right),
\end{gathered}
$$

$$
\begin{gathered}
\pi_{R}=O(r), \\
\pi_{\gamma}=O\left(r^{2}\right), \\
\pi_{\psi}=O(r), \\
\pi_{\tilde{\psi}}=\pi_{\tilde{\psi}}(0)+O(r) .
\end{gathered}
$$

The lapse and shift are assumed to have the following behavior as $r \rightarrow 0$ :

$$
\begin{gathered}
N^{\perp}=N^{\perp}(0)+O\left(r^{2}\right), \\
N^{r}=O\left(r^{2}\right) .
\end{gathered}
$$

The lapse density inherits its behavior as $r \rightarrow 0$ from the lapse function:

$$
N=N(0)+O\left(r^{2}\right) .
$$

Equations (A6) and (3.4) imply that as $r \rightarrow 0$,

$$
T=T(0)+O\left(r^{3}\right) .
$$

Similarly, we assume

$$
\Pi_{T}=O(r)
$$

and

$$
\Pi_{R}=O(r) .
$$

Our boundary conditions at $r=0$ imply that there are no singularities on the axis of symmetry.

As $r \rightarrow \infty$, we assume the following behavior of the canonical coordinates and momenta on $\Gamma$ :

$$
\begin{gathered}
R=r+O\left(r^{-\epsilon}\right), \\
\gamma=\gamma(\infty)+O\left(r^{-\epsilon}\right), \\
\psi=O\left(r^{-\epsilon}\right), \\
\widetilde{\psi}=O\left(r^{-\epsilon}\right), \\
\pi_{R}=O\left(r^{-1}\right), \\
\pi_{\gamma}=O\left(r^{-(1+\epsilon)}\right), \\
\pi_{\psi}=O\left(r^{-1}\right), \\
\pi_{\psi}=O\left(r^{-(1+\epsilon)}\right),
\end{gathered}
$$

where $\epsilon>0$. The lapse and shift are assumed to have the following behavior as $r \rightarrow \infty$ :

$$
\begin{gathered}
N^{\perp}=N^{\perp}(\infty)+O\left(r^{-\epsilon}\right), \\
N^{r}=O\left(r^{-\epsilon}\right) .
\end{gathered}
$$

The lapse density inherits its behavior as $r \rightarrow \infty$ from the lapse function:

$$
N=N(\infty)+O\left(r^{-\epsilon}\right) .
$$

Equations (A20) and (3.4) imply that, as $r \rightarrow \infty$, 


$$
T=T(\infty)+O\left(r^{-\epsilon}\right) .
$$

and

Similarly,

$$
\Pi_{T}=O\left(r^{-(1+\epsilon)}\right)
$$

$$
\Pi_{R}=O\left(r^{-1}\right)
$$

[1] K. V. Kuchař, in General Relativity and Relativistic Astrophysics, Proceedings of The Fourth Canadian Conference on General Relativity and Relativistic Astrophysics, Winnipeg, Canada, 1991, edited by G. Kunstatter, D. Vincent, and J. Williams (World Scientific, Singapore, 1992).

[2] R. Arnowitt, S. Deser, and C. Misner, in Gravitation: An Introduction to Current Research, edited by L. Witten (Wiley, New York, 1962).

[3] P. A. M. Dirac, Proc. R. Soc. London A246, 333 (1958).

[4] Y. Choquet-Bruhat and J. York, in General Relativity and Gravitation: 100 Years After the Birth of Albert Einstein, edited by A. Held (Plenum, New York, 1980), Vol. 1.

[5] P. A. M. Dirac, Lectures on Quantum Mechanics (Yeshiva University, New York, 1964).

[6] K. V. Kuchař, J. Math. Phys. 13, 768 (1972).

[7] K. V. Kuchar, Phys. Rev. D 4, 955 (1971).

[8] D. E. Neville, Class. Quantum Grav. 10, 2223 (1993).

[9] K. V. Kuchař, Phys. Rev. D 50, 3961 (1994).

[10] K. V. Kuchař and C. G. Torre, J. Math. Phys. 30, 1769 (1989).

[11] K. V. Kuchař and C. G. Torre, in Conceptual Problems of Quantum Gravity, edited by A. Ashtekar and J. Stachel (Birkhäuser, Boston, 1991).

[12] C. G. Torre, Phys. Rev. D 40, 2588 (1989).

[13] T. Regge and C. Teitelboim, Ann. Phys. (N.Y.) 88, 286 (1974).

[14] C. G. Torre, Phys. Rev. D 46, R3231 (1992).
[15] R. Gowdy, Ann. Phys. (N.Y.) 83, 203 (1974).

[16] R. Gowdy, Phys. Rev. Lett. 27, 826 (1971); 27, 1102 (1971).

[17] R. Geroch, J. Math. Phys. 12, 918 (1971).

[18] R. Geroch, J. Math. Phys. 13, 394 (1971).

[19] B. Berger, P. Chruściel, and V. Moncrief, Ann. Phys. (N.Y.) 237, 322 (1995).

[20] A. Ashtekar and M. Varadarajan, Phys. Rev. D 50, 4944 (1994).

[21] C. Teitelboim, in Quantum Theory of Gravity, edited by S. Christensen (Hilger, Bristol, 1984).

[22] This Lie algebra is isomorphic to the Lie algebra of the twodimensional conformal group. See [10] for an interpretation of this result.

[23] V. Moncrief, Ann. Phys. (N.Y.) 132, 87 (1980).

[24] This strategy can be viewed as a field theoretic generalization of a proposal made by Hajicek in a finite-dimensional context; see P. Hajicek, Phys. Rev. D 38, 3639 (1988).

[25] K. V. Kuchař and C. G. Torre, Phys. Rev. D 43, 419 (1991).

[26] It is possible to translate the transformation (3.86)-(3.91) into a form applicable to the cylindrically symmetric theory, but in this case the boundary conditions needed on the harmonic maps are somewhat unnatural.

[27] K. Kuchar, Phys. Rev. D 39, 2263 (1989).

[28] M. Henneaux and C. Teitelboim, Quantization of Gauge Systems (Princeton University Press, Princeton, NJ, 1992). 